\title{
Syringol Isolated From Eleusine Coracana (L.) Gaertn Bran Suppresses Inflammatory Response Through the Down-Regulation of CPLA2, COX-2, IKBa, p38 and MPO Signaling in SPLA2 Induced Mice Paw Edema.
}

Mallanayakanakatte D. Milan Gowda

University of Mysore

Jayachandra K

University of Mysore

Vikram Joshi

Mayo Clinic Division of Gastroenterology and Hepatology

Vaddarahally N. Manjuprasanna

University of Mysore

Gotravalli V. Rudresha

University of Mysore

Devadasan Velmurugan

University of Madras - Guindy Campus

Raman Pachaiappan

SRM Institute of Science and Technology

Noor Mohamed Jameel

University of Mysore

Bannikuppe S. Vishwanath ( $\square$ vishmy@biochemistry.uni-mysore.ac.in )

University of Mysore https://orcid.org/0000-0003-3284-4163

Research Article

Keywords: Eleusine coracana, arachidonic acid, secretory phospholipase A2, lipoxygenase, cyclooxygenase and inflammation.

Posted Date: July 29th, 2021

DOI: https://doi.org/10.21203/rs.3.rs-755579/v1 
License: (c) (i) This work is licensed under a Creative Commons Attribution 4.0 International License. Read Full License 
Syringol isolated from Eleusine coracana $(L$. $)$ Gaertn bran suppresses inflammatory response through the down-regulation of $\mathrm{CPLA}_{2}, \mathrm{COX}-2, \mathrm{I} \mathrm{I} B \alpha, \mathrm{p38}$ and MPO signaling in $\mathrm{SPLA}_{2}$ induced mice paw edema.

Mallanayakanakatte D. Milan Gowda a, Jayachandra K a, Vikram Joshi b, Vaddarahally N. Manjuprasanna a, Gotravalli V. Rudresha a, Devadasan Velmurugan ${ }^{\mathrm{c}}$, Raman Pachaiappan ${ }^{\mathrm{d}}$, Noor Mohamed Jameel $^{\text {a }}$, Bannikuppe S. Vishwanath ${ }^{\text {* }}$

a Department of Studies in Biochemistry, University of Mysore, Manasagangotri, Mysuru, Karnataka, India.

b Division of Gastroenterology \& Hepatology, Enteric NeuroScience Program , Mayo Clinic, Rochester, Minnesota, USA.

c Centre of Advanced Study in Crystallography and Biophysics, University of Madras, Chennai, Tamil Nadu, India.

d Department of Biotechnology, School of Bioengineering, SRM Institute of Science and Technology, Kattankulathur, Tamil Nadu, India.

\section{* Corresponding author}

Dr. B. S. Vishwanath

Tel.: +91-9845893634

E-mail: vishmy@biochemistry.uni-mysore.ac.in

Dr. Noor Mohamed Jameel

Tel.: +91-9880378780

E-mail: jameel.nm@gmail.com

Abbreviations: $\mathrm{SPLA}_{2}$ - Secretory phospholipase $\mathrm{A}_{2}$; AA - Arachidonic acid: COX - Cycloxygenase; LOX Lipoxygenase; HSF - Human synovial fluid; Ecb - E coracana bran; Ecsp - E coracana seed powder. 


\begin{abstract}
Eleusine coracana (L.) Gaertn (E. coracana) is one of the highest consuming food crops in Asia and Africa. E. coracana is a plant with several medicinal values including anti-ulcerative, anti-diabetic, anti-viral and anticancer properties. However, the anti-inflammatory property of E. coracana remains to be elucidated. Therefore, the objective of present study was to investigate the potential in isolated molecule from E. coracana via a combination of in vitro, in vivo and in silico methods. In this study we have isolated, purified and characterized an anti-inflammatory molecule from E coracana bran extract known as syringol. Purification of syringol was accomplished by combination of GC-MS and RP-HPLC techniques. Syringol significantly inhibited the enzyme activity of $\operatorname{sPLA}_{2}\left(\mathrm{IC}_{50}=3 \mu \mathrm{g}\right)$ and 5 -LOX $\left(\mathrm{IC}_{50}=0.325 \mu \mathrm{g}\right)$ in vitro. The inhibition is independent of substrate concentration, calcium ion concentration and was irreversible. Syringol interacts with purified sPLA enzymes $_{2}$ as evidenced by fluorescence and molecular docking studies. Further, the syringol molecule dose dependently inhibited the development of $\mathrm{sPLA}_{2}$ and carrageenan induced edema. Furthermore, syringol decreases the expression of $\mathrm{cPLA}_{2}, \mathrm{COX}-2, \mathrm{I} \kappa \mathrm{B} \alpha, \mathrm{p} 38$ and MPO in edematous tissues as demonstrated by western blots. These studies revealed that syringol isolated from E coracana bran may develop as a potent anti-inflammatory molecule.
\end{abstract}

Key words: Eleusine coracana, arachidonic acid, secretory phospholipase $\mathrm{A}_{2}$, lipoxygenase, cyclooxygenase and inflammation.

\title{
Abbreviations:
}

sPLA $_{2} \quad$ Secretory phospholipase $A_{2}$

HSF-PLA $_{2} \quad$ Human synovial fluid phospholipase $\mathrm{A}_{2}$

NN-PLA $2 \quad$ Naja naja phospholipase $\mathrm{A}_{2}$

AA Arachidonic acid

COX Cyclooxygenase

LOX Lipoxygenase (LOX)

Ecb E. coracana bran

Ecsp Seed powder

EBME Eleusine coracana bran methanol extract 


\section{Introduction}

Inflammation is a critical self-protective response to multiple stimuli in response to body's fight against invading pathogens, infection, injury and cell damage (Medzhitov 2010). The initiator enzyme that triggers inflammatory reactions is secretory phospholipase $\mathrm{A}_{2}\left(\mathrm{sPLA}_{2}\right)$, acts at the $s n-2$ position of membrane phospholipids to release arachidonic acid (AA). The released AA is oxidized by cyclooxygenase (COX) and lipoxygenase (LOX) enzymes to produce various pro-inflammatory mediators called eicosanoids (Mirzoeva and Calder 1996, Liu, Tang et al. 2019). Targeting a single enzyme of eicosanoid pathway is not an ideal strategy because other active pro-inflammatory enzymes in system can still elicit the inflammatory reactions. Therefore, we emphasize that cumulative inhibition of $\mathrm{sPLA}_{2}, 5-\mathrm{LOX}$ and COX-2 activity by a single molecule is of great therapeutic importance (Yedgar, Lichtenberg et al. 2000, Joshi, Umashankara et al. 2016).

Currently, several synthetic drugs such as non-steroidal anti-inflammatory drugs (NSAIDs) and glucocorticoids are limited to treat several debilitating inflammatory conditions (Tarayre and Lauressergues 1979). Although these treatment options are effective in improving disease conditions, chronic and prolonged use of these agents are reported with several ill effects such as gastric ulceration, bleeding, and emergence of opportunistic infections due to immunosuppression (Laine 2003). In consideration with human health, researchers are engage in the search of disease preventive phenolic compounds in routinely used food crops due to its therapeutic role in regulation of various diseases conditions (Zhu, Du et al. 2018, Forni, Facchiano et al. 2019). . In addition, phenolic compounds are reported to regulate the inflammatory enzymes such as sPLA, LOX and COX-2, thereby preventing inflammation (Rao, Rivenson et al. 1995, Rao, health et al. 2007). Therefore, identifying and characterizing a novel, more potent, less harmful molecule with anti-inflammatory properties has the potential to gain considerable attention in the field of medicine.

Eleusine coracana (E. coracana) (Family: Poaceae) commonly known as finger millet, nutritionally rich routinely used food crops in most parts of tropics and subtropics of Asia and Africa (Chandra, Chandra et al. 2016, Kumar, Metwal et al. 2016). E. coracana is also used as medicinal plant due to its biological activities such as anti-diabetic, anti-cancer, anti-viral properties, also used to treat constipation, leprosy, liver disease, measles and pneumonia (Luitel, Siwakoti et al. , Breyer-Brandwijk, Southern et al. 1962, Chethan and Malleshi 2007, Srivastava, Sharma et al. 2012). During the processes of milling, bran is removed and seed devoid of bran is used for the consumption (Jun, Song et al. 2012). E. coracana bran is presumed to be less nutritional, used as a fodder for animals (Budhwar, Chakraborty et al. 2020). However, bran of other species from Poaceae family 
such as Oryza sativa (O. sativa) and Sorghum bicolor (S. bicolor) is rich in medicinally important phytochemicals. which are reportd to possess anti-inflammatory, anti-oxidant, anti-diabetic, anti-cancer, antidiabetic potentials and mouse paw edema curative properties (Awika, Rooney et al. 2003, Burdette, Garner et al. 2010, Kozuka, Sunagawa et al. 2015, Suttiarporn, Chumpolsri et al. 2015, Somintara, Leardkamolkarn et al. 2016, Ramazani, Akaberi et al. 2020). Since, E. coracana, O. sativa, and S. bicolor belong to the same family, Poaceae, and based on the above studies, we suspected that E. coracana bran could also be therapeutically important but to the best of our knowledge not much information is available, except a study which shows $E$. coracana bran purified molecule arabinoxylan have immunomodulatory activity (Savitha Prashanth, Shruthi et al. 2015). With reference to available research findings, the present study aimed to investigate the therapeutic potential of E. coracana bran with an emphasis on its anti-inflammatory properties. We have successfully isolated, characterized and evaluated a purified phytochemical with anti-inflammatory properties from $E$. coracana bran. The isolated compound has been systematically evaluated for its effectiveness towards inhibition of pro-inflammatory sPLA 2 , COX-2 and 5-LOX enzymes and in vivo anti-inflammatory activity.

\section{Materials and methods}

\subsection{Chemicals and reagents}

Ketamine and xylocaine were purchased from the University Medical Facility with a prescription from the University authorized medical practitioner. 4-nitro-3-octanoyloxy-benzoic acid was purchased from abcam Cambridge, MA, USA. Syringol, $\lambda$-carrageenan and HPLC grade solvents were purchased from Sigma-Aldrich, St. Louis, USA. Bovine serum albumin (BSA), ethylene diamine tetraacetic acid (EDTA), Dimethylsulphoxide (DMSO), and calcium chloride were purchased from Sisco Research Laboratories (SRL) Pvt. Ltd. (Mumbai, India). Antibodies against p-cPLA 2 (1:1000), p-COX-2 (1:1000), p-IkB $\alpha$ (1:1000), p-P38 (1:1000), MPO (1:1000) and $\beta$-actin (1:5000) were purchased from Cell Signalling Technology (Massachusetts, USA). HRP tagged goat anti-rabbit antibody (1:10000) was procured from Jackson Immuno Research (Philadelphia, USA). Polyvinylidene difluoride (PVDF) membrane was purchased from Merck Millipore (Darmstadt, Germany). All the other chemicals and reagents used in this study were of analytical grade. Solvents were redistilled before use.

\subsection{Experimental animals}

Male Swiss albino mice weighing 20 to 25 g (6 to 8-weeks old; both genders) were obtained from Central Animal House Facility, Department of Studies in Zoology, University of Mysore, Mysuru, India. The 
animal care and handling were conducted in compliance with National Regulations for Animal Research. The animal experiments were carried out after the approval of the protocols by the Animal Ethics Committee of the University of Mysore, Mysuru, India (Order No: UOM/IAEC/13/2018).

\section{Methods:}

\subsection{Extraction, phytochemical analysis and isolation of phenol rich extract from E coracana bran}

Briefly 20 g of dry E. coracana bran (Ecb) and seed powder (Ecsp) were subjected to hot extraction using soxhlet apparatus separately according to the method of William B. Jensen et al. with some modifications (Jensen 2007). Extraction was done by using different solvents with increasing polarity (n-hexane, ethyl acetate, methanol and water). The extracts obtained from these solvents were filtered and dried completely in flash evaporator and the dry weight was determined. The extracts were re-dissolved in suitable solvents and serially diluted in phosphate buffer for further use. Later the hot extracts of Ecb and Ecsp extracts were screened qualitatively for different phytochemicals using standard procedures as described (Verma and Singh 2020).

Further, phenol rich extract (PE) was isolated from E coracana bran methanol extract (EBME) by adopting standardized procedure (Nanjaraj Urs, Yariswamy et al. 2015). Briefly, EBME (200 mg/ml) was suspended in acidified methanol. The acidified sample was flash evaporated, re-suspended in $20 \mathrm{ml}(20 \%)$ ethanol and the content was mixed with $25 \mathrm{ml}$ of ethyl acetate in a separating funnel. The collected upper phase (ethyl acetate) was rehydrated in sodium sulphate. Finally, the obtained PE was flash evaporated and resuspended in $2 \mathrm{ml} \mathrm{(50 \% )} \mathrm{methanol} \mathrm{and} \mathrm{filtered} \mathrm{through} 0.45$ micron pore-size syringe filter for RP-HPLC and GC-MS analysis.

\subsection{GC-MS analysis}

GC MS (Agilent, USA), coupled with a (7890B- GC and 5977A MSD) Agilent mass selective detector (Triple-Axis Detector) was used for the analysis. The GC-MS system was equipped with a HP-5MS $5 \%$ Phenyl methyl silox column (30 m x $250 \mu \mathrm{m} \times 0.25 \mu \mathrm{m}$ film thickness). Analyses were carried out using helium as carrier gas at a flow rate of $1 \mathrm{ml} / \mathrm{min}$ and a split ratio of 1:1 using the following conditions, $95 \mathrm{~min}$ total and run time temperature program: program starts with $50{ }^{\circ} \mathrm{C}$ for $2 \mathrm{~min}$ hold, then ramped $3{ }^{\circ} \mathrm{C} / \mathrm{min}$ up to $270{ }^{\circ} \mathrm{C}$, then hold for $20 \mathrm{~min}$ at same temperature. $1 \mu \mathrm{l}$ of diluted sample was injected to column and mass spectra were obtained by electron impact ionization $70 \mathrm{eV}$ at scan range $40-700 \mathrm{~m} / \mathrm{z}$. Compounds were identified by matching of their mass spectra with NIST library. 


\subsection{RP-HPLC profiling of EBME}

HPLC of phenolics was carried out using Waters, Waltham, MA. Phenolics purification was performed according to (Cantin, Moreno et al. 2009) with mobile phase consisting of methanol: acetic acid: water (80:2:18; $\mathrm{v} / \mathrm{v} / \mathrm{v})$. The mobile phase was pumped with a Waters 515 HPLC pump at a flow rate of $0.5 \mathrm{ml} / \mathrm{min}$ for $60 \mathrm{~min}$. $20 \mu 1$ of sample was injected in to pre-equilibrated Varian Microsorb column (C18, $5 \mu, 4.6$ x $250 \mathrm{~mm})$ and phenolics were detected using Waters $2489 \mathrm{UV}$-Vis detector at $270 \mathrm{~nm}$. Standard phenolic acids such as gallic acid, catechin, caffeic acid, chlorogenic acid, p-coumaric acid, ferulic acid, epicatechin, protocatechuic acid, syringol, vanillic acid and trans-cinnamic acid were used.

\subsection{Source of SPLA}

sPLA $_{2}$ from Naja naja and Daboia russelii venom was partially purified as described in our previous publication (Joshi, Venkatesha et al. 2016). sPLA $_{2}$ from human synovial fluid (HSF) was obtained by DRM multi-specialty hospital, Mysuru and human pleural fluid (HPF) was obtained from Princess Krishnarajamani Tuberculosis (PKTB) Hospital, Mysuru, India. $\mathrm{SPLA}_{2}$ from HSF and HPF were purified according to our previously published protocol (Franson, Raghupathi et al. 1990, Jameel, Frey et al. 2005). All experiments involving human biological samples were conducted according to the protocols approved by the Institutional Human Ethics Committee, UOM, Mysuru (Sanction order no: IHEC-UOM No.136/Ph.D/2016-17) and were in accordance with the regulations of the Indian Council of Medical Research, New Delhi, India

\subsection{Phospholipase $A_{2}$ assay and its inhibition}

sPLA $A_{2}$ activity assay was carried out in 96-well plate according to the method of W H Lee et al. with slight modifications (Lee, Toyama et al. 1999). Briefly, the standard assay mixture contained $200 \mu 1$ of buffer (10 mM Tris- $\mathrm{HCl}, 10 \mathrm{mM} \mathrm{CaCl}_{2}, 100 \mathrm{mM} \mathrm{NaCl}, \mathrm{pH} 7.8$ ), $60 \mathrm{mM}$ of substrate (4-nitro-3-octanoyloxy-benzoic acid), $20 \mu \mathrm{l}$ of water and $30 \mu \mathrm{g}$ of $\mathrm{sPLA}_{2} \mathrm{~s}$ adding to a final volume of $260 \mu \mathrm{l}$. After the addition of $\mathrm{SPLA}_{2} \mathrm{~s}$, the mixture was incubated for up to $40 \mathrm{~min}$ at $37{ }^{\circ} \mathrm{C}$, its absorbance at $425 \mathrm{~nm}$ being read at 10 min intervals. Enzyme activity was calculated based on the increase in absorbance after 20 min. For inhibition studies, extracts and syringol was pre-incubated with enzyme for 15 min at $37{ }^{\circ} \mathrm{C}$ separately and $\mathrm{sPLA}_{2}$ activity was measured and the results were expressed as \% residual activity. To evaluate the effect of substrate and calcium concentration on inhibition of $\mathrm{sPLA}_{2}$, the experiment was performed with varying concentration of substrate and calcium in the presence of syringol $(3 \mu \mathrm{g})$. The activity of enzyme alone was considered as $100 \%$ activity and enzyme inhibition was expressed in terms of \% residual activity. 


\subsection{Neutralization of secretory phospholipase $A_{2}$ induced mouse paw edema}

Mouse paw edema was induced as described by Vishwanath et al. (Vishwanath, Fawzy et al. 1988) was followed to induce paw edema. Briefly, mice ( $\mathrm{n}=3$ ) were given intraplantar injection with $40 \mu \mathrm{l}$ of saline containing $4 \mu \mathrm{g}$ of $\mathrm{sPLA}_{2}$ (HSF and Naja naja $\mathrm{PLA}_{2}$ ) to the right mice footpad. Respective left footpad was injected with $40 \mu \mathrm{l}$ of saline/vehicle which served as a negative control/vehicle control. After $45 \mathrm{~min}$, mice were euthanized by using anaesthetic pentobarbitone (30 mg/kg; i.p.), both hind limbs were excised at the ankle joint and weighed individually. Animals treated with $\mathrm{SPLA}_{2}$ were served as positive control. For inhibition studies 4 $\mu \mathrm{g}$ of sPLA $\mathrm{A}_{2}$ was pre-incubated with or without syringol and vehicle for $15 \mathrm{~min}$ at $37{ }^{\circ} \mathrm{C}$ was co-injected to right paw and respective left paw received saline/vehicle. The edema ratio was calculated using the formula;

$$
\text { Edema ratio }=(\text { Weight of edematous leg } / \text { Weight of normal leg }) \times 100
$$

\subsection{Western blotting}

Edematous tissues from respective groups of mice were excised on 45 minutes after intraplantar injection. Edema tissue samples were snap-frozen in liquid nitrogen and homogenized. The homogenized tissues were kept in $-20{ }^{\circ} \mathrm{C}$ for overnight in EDTA-free cold lysis buffer containing protease inhibitor mixture. Tissue homogenates were centrifuged at $9000 \mathrm{~g}$ for $10 \mathrm{~min}$ and the supernatant was used for protein quantification (Lowry, Rosebrough et al. 1951). Briefly, $30 \mu \mathrm{g}$ of tissue lysate was separated by SDS-PAGE and electrotransferred to a PVDF membrane. Further, the membranes were blocked with $5 \%$ BSA in TBST (20 mM Tris, pH 7.5 with $150 \mathrm{mM} \mathrm{NaCl}$ and $0.05 \%$ Tween-20). Then, the blots were incubated with primary antibody; pcPLA2, pCOX-2, pIkB $\alpha$, MPO and $\beta$ - actin overnight at $4{ }^{\circ} \mathrm{C}$ as per manufacturer's recommendation. Following TBST wash, blots were probed with HRP conjugated anti-rabbit or anti-mouse IgG (1:10000) secondary antibody for $1 \mathrm{~h}$ at room temperature. Further, blots were washed with TBST, developed by enhanced chemiluminescence and visualized using a Bioimaging system (Alliance 2.1 UVITEC Cambridge image analyzer, UK). $\beta$-actin was used as a loading control.

\subsection{Computational studies}

To understand the molecular binding patter of syringol with crystal structure of inflammatory targets such as $N$. naja $\mathrm{sPLA}_{2}$ (PDB ID: 2WQ5), Human inflammatory exudate sPLA 2 (PDB ID: 1POE), D. russelii sPLA $_{2}$ (PDB ID: 3H1X), sheep COX-1 (PDB ID: 3N8W), mouse COX-2 (PDB ID: 3NT1) and human mutant 5LOX (PDB ID: 3O8Y) were employed by the induced fit docking (IFD) analysis. The crystal structures of 
inflammatory targets were retrieved from protein data bank (PDB). The modeller software was used through the Chimera interface to model the 3D structures of 5-LOX, COX-1 and COX-2. Human 5-LOX structure was modelled using the structure of human mutant 5-LOX as template. Similarly, human COX-1 and COX-2 were modelled using the structure of sheep COX-1 and mouse COX-2 as template respectively. The discrete optimized potential energy (DOPE) and Ramachandran plot were used to validate the model selected for docking. The structure (crystal/homology) of the proteins were prepared using "Protein preparation wizard" of the Schrodinger software as it facilitates assignment of proper bond order, addition of charges, and protonation state prior to minimization. Hydrogen bond network optimization and identification of proper ionization states (His tautomers) were performed. Terminal angle of Asn, Gln, and His residues was allowed for $180{ }^{\circ}$ rotations. Impref module was used for final constrained energy minimization with root mean square deviation (RMSD) cut-off of $0.30 \AA$. The active site amino acids of the modelled proteins or the co-crystallized ligand in the crystal structure were used to locate the active site and the compounds of interest were docked. The structure of the ligand molecule syringol was retrieved from the Pubchem structure database in 3D SDF file. All the ligands were prepared using the ligprep module. $\mathrm{pH} 7 \pm 2$ was set for ionization. Maximum of 32 stereo isomers and tautomers were allowed to generate conformational state of each ligand. Chiralities of stereoisomers and low energy ring conformation were retained. IFD method facilitates introducing flexibility in both ligand as well as the protein during docking by combining the Glide docking and the prime structure refinement methods, with several iterations. For each ligand docking, maximum of 20 poses were obtained and the best of them was chosen based on the glide score, energy and the interactions with the active site residues. The OPLS_2005 force field was used for the calculations involved in ligand and protein preparations and docking using Schrodinger software package. The physicochemical properties of syringol were calculated using Qikprop module.

\subsection{Statistical analysis}

The results were expressed as mean \pm SD. One-way ANOVA with Bonferroni's multiple comparison post hoc tests was performed to assess statistical significance using Graph Pad Prism 5.0 software. The comparison between the groups was considered significant if $\mathrm{p}<0.05$.

\section{Results:}

\subsection{Extraction and qualitative analysis of phytochemicals from E. coracana}

The extraction and analysis of phytochemicals of E. coracana bran (Ecb) and E. coracana seed powder (Ecsp) were performed as described in methods section. The qualitative analysis reveals the presence of 
phenolics, flavonoids, terpenoids and glycosides. High levels of phenolics $(2.0 \%)$ and saponins $(0.9 \%)$ were observed in Ecb methonolic extracts whereas saponins, glycosides and alkaloids were found in Ecsp methonolic extract (Supplementary Table 01). The yield of different solvent extracts of Ecb and Ecsp were shown in Supplementary Table 02. The total recovery concentration of phytochemical was more in seed powder extracts $(8.25 \%)$ compared to bran extracts $(1.75 \%)$.

\begin{tabular}{|c|c|c|c|c|c|c|c|c|}
\hline \multirow{2}{*}{ Phytochemicals } & \multicolumn{4}{|c|}{ E. coracana bran } & \multicolumn{4}{|c|}{ E. coracana seed powder } \\
\hline & Hexane & $\begin{array}{c}\text { Ethyl } \\
\text { acetate }\end{array}$ & Methanol & Water & Hexane & $\begin{array}{c}\text { Ethyl } \\
\text { acetate }\end{array}$ & Methanol & Water \\
\hline Alkaloids & - & - & - & - & - & - & ++ & + \\
\hline Anthraquinone & - & - & - & - & - & - & - & - \\
\hline Flavonoids & - & - & - & - & - & - & + & - \\
\hline Glycosides & - & + & - & + & - & + & ++ & + \\
\hline Phenolics & - & + & +++ & ++ & - & - & - & - \\
\hline Saponins & - & - & ++ & - & ++ & - & +++ & - \\
\hline Steroids & - & - & - & - & + & - & - & - \\
\hline Tannins & - & - & - & - & - & - & - & - \\
\hline Terpenoids & - & - & - & - & ++ & + & - & - \\
\hline
\end{tabular}

Supplementary Table 01: Phytochemical analysis of $\boldsymbol{E}$. coracana bran and seed powder: The E. coracana bran and seed powder was subjected to hot extraction using solvents with increasing polarity (n-hexane, ethyl acetate, methanol and water). After extraction, solvents were evaporated and weight of extracts was recorded and re-constituted in respective solvents and used for further analysis.

\begin{tabular}{lcc}
\hline \multicolumn{1}{c}{ Solvents } & Ecb $(\%$ yield $)$ & Ecsp $(\%$ yield $)$ \\
\hline Hexane & 0.10 & 1.10 \\
Ethyl acetate & 0.15 & 1.60 \\
Methanol & 0.30 & 2.15 \\
Water & 1.20 & 3.40 \\
\hline
\end{tabular}

Supplementary Table 02: The yield of phytochemicals in different solvent extracts of (Ecb) and (Ecsp).

\subsection{In vitro and in vivo anti-inflammatory activities of Ecb and Ecsp crude extracts}

To investigate the in vitro anti-inflammatory potential, Ecb and Ecsp crude extracts were screened for ${ }_{\text {sPLA }}$ enzyme inhibitory activity. Among all the extracts, E coracana bran methanol extract (EBME) 
significantly inhibited the Naja naja (N. naja) and human synovial fluid (HSF) sPLA 2 activity (Fig. 1a \& b). The inhibition was concentration dependent and significant inhibition was observed at $25 \mu \mathrm{g}$ of EBME (Fig. 1c). Based on this finding, the EBME was further used to demonstrate the in vivo anti-inflammatory activity.

Animals injected with $4 \mu \mathrm{g}$ of human synovial fluid sPLA $_{2}\left(\mathrm{HSF}_{\mathrm{sPLA}}\right)$ into hind paw developed swelling of footpad and the calculated edema ratio is $165.5 \pm 4.94 \%$ considered as $100 \%$. However, animals co-injected with EBME and $\mathrm{sPLA}_{2}$ the edema ratio was dose dependently reduced up to $89 \pm 5.65 \%$ (Fig. 1d). These findings indicate the presence of anti-inflammatory molecules in EBME.
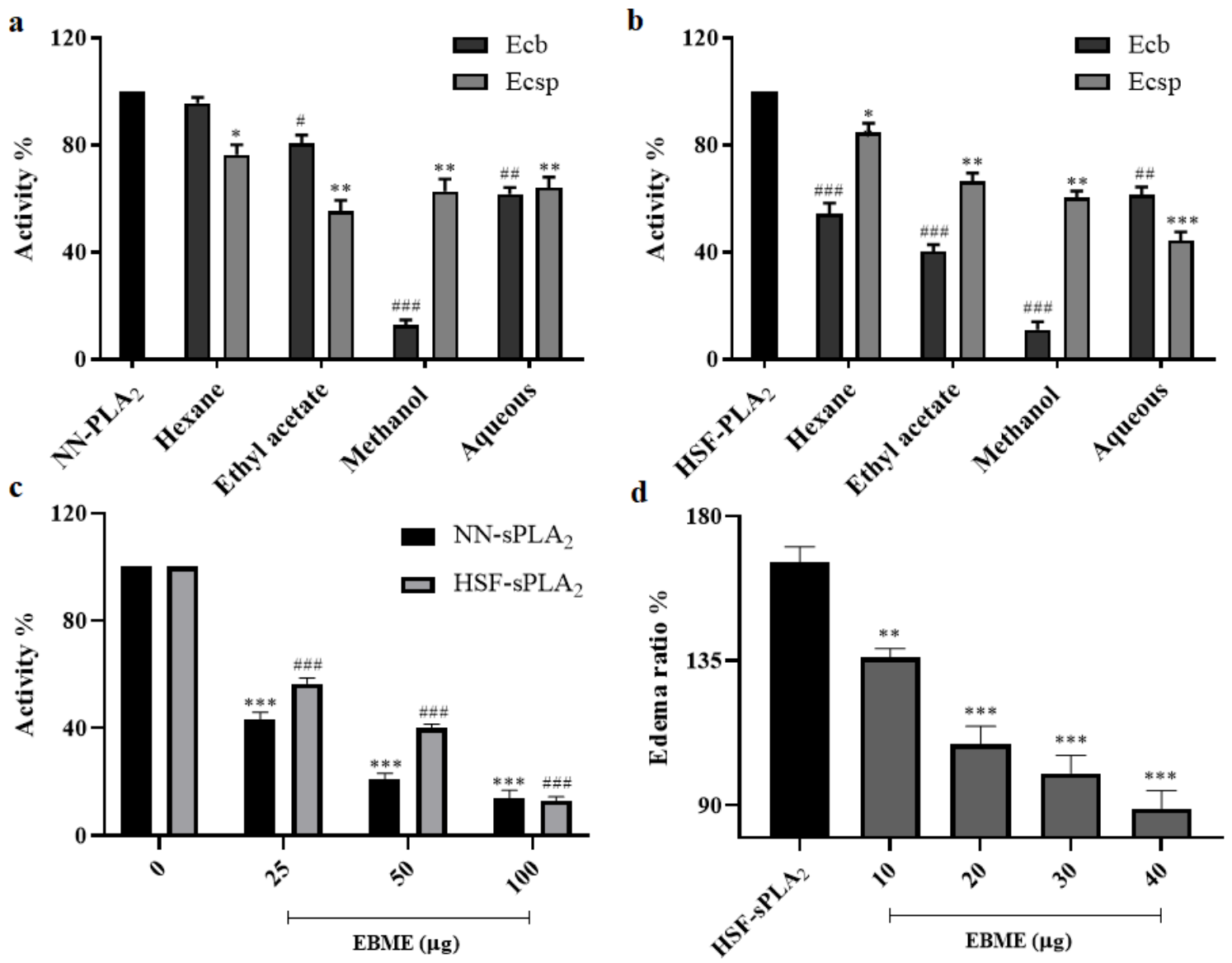

Fig. 1. Inhibition of $\mathbf{S P L A} \mathbf{A}_{2}$ activity by Ecb and Ecsp extracts. (a $\&$ b) $\operatorname{sPLA}_{2}(30 \mu \mathrm{g})$ was pre-incubated with $100 \mu \mathrm{g}$ of Ecb and Ecsp extracts for $15 \mathrm{~min}$. The reaction was initiated by adding $200 \mu \mathrm{l}$ of standard assay mixture, $20 \mu \mathrm{l}$ of substrate, $20 \mu \mathrm{l}$ of water and $20 \mu \mathrm{l}$ of $\mathrm{sPLA}_{2}$. The mixture was incubated for $40 \mathrm{~min}$ at $37^{\circ} \mathrm{C}$ and absorbance was recorded at $425 \mathrm{~nm}$. (c) Different doses of EBME were pre-incubated with $30 \mu \mathrm{g}$ of sPLA 2 in standard assay conditions and absorbance was record at $425 \mathrm{~nm}$. (D) $4 \mu \mathrm{g}$ of HSF-sPLA 2 with or without different dose of EBME in a total reaction volume of $40 \mu \mathrm{l}$ and injected into mice right footpad. Where left 
footpad receive $40 \mu 1$ of saline and vehicle. Data represents Mean $\pm \operatorname{SD}(\mathrm{n}=3) .{ }^{*}, \#<0.05,{ }^{* *}, \# \# \mathrm{p}<0.01$ and ${ }^{* * *}$, $\# \#$ p $<0.001$ as compared to $\mathrm{sPLA}_{2}$ alone.

\subsection{Purification of phenolic compound from EBME}

The preliminary data obtained encouraged us to carry out further purification of active molecule from EBME. Phenol rich extract (PE) from EBME was isolated as described in the methods (Supplementary Fig. 1). The GC-MS analysis of PE led to identify some compounds (Fig 2). The chromatogram peaks were integrated and compared with GC-MS data base of known compounds confirms the presence of syringol in PE. Among other identified compounds in PE, syringol is the only uncharacterized phenolic compound with antiinflammatory activities. We have listed all the identified compounds in Table 1. The same PE was injected into RP-HPLC and it resolved into a major peak with few minor peaks. From the obtained chromatogram we found that the retention time of major peak was 7.034 min (Fig. 3a) which is equivalent to the standard syringol retention time 7.177 min (Supplementary Fig 2). Amongst the collected RP-HPLC peaks, the major peak confirmed the sPLA 2 inhibitory activity. Purity of syringol isolated from PE was verified by RP-HPLC (Fig. 3b). Inhibition of $\mathrm{PLA}_{2}$ enzyme by EBME and PE along with purified syringol from Ecb is represented in

\section{Supplementary Fig 3.}


The E. coracana (ragi) bran $200 \mathrm{mg} / \mathrm{ml}(\mathrm{w} / \mathrm{v})$ was extracted with methanol and acidified. The acidified crud extract pellet was re-suspended in $20 \%(\mathrm{v} / \mathrm{v})$ ethanol after rotary evaporator.

The ethanol solubilized fraction was mixed with ethyl acetate and allowed to phase separation in separating funnel.

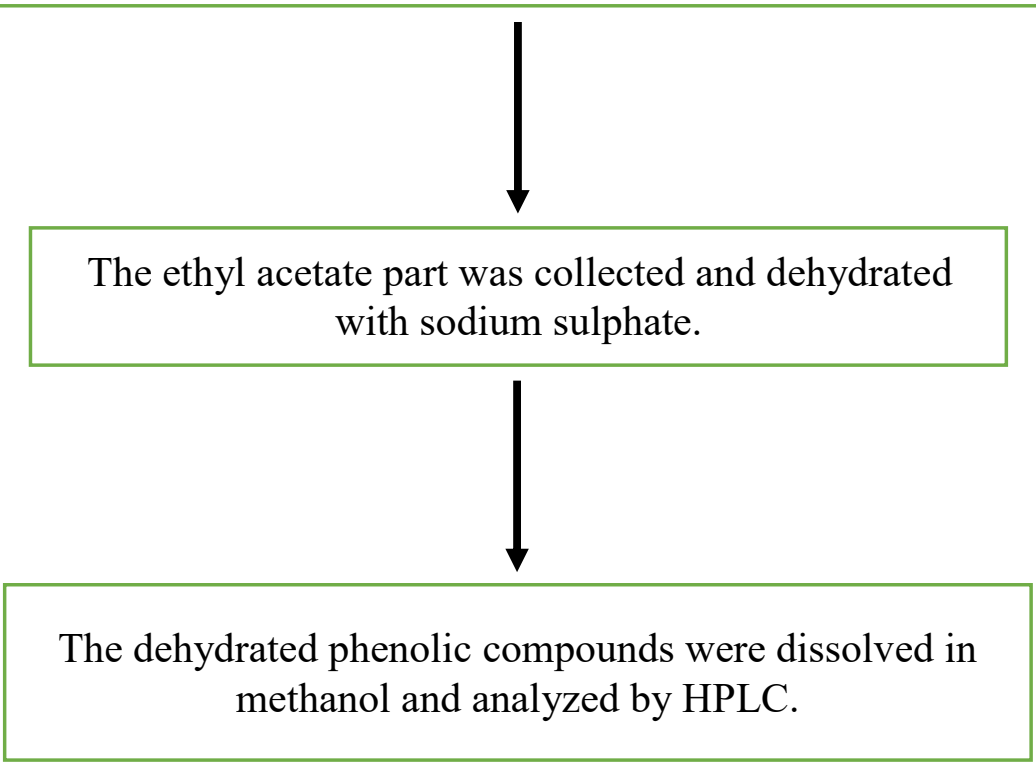

Supplementary Fig 1. The schematic representation for extraction of PE from EBME. $200 \mathrm{mg}$ of EBME was subjected to acid hydrolysis, then treated with ethanol and separated in ethyl acetate, dehydrated with sodium sulphate and dissolved in methanol and subjected to HPLC fractionation. 


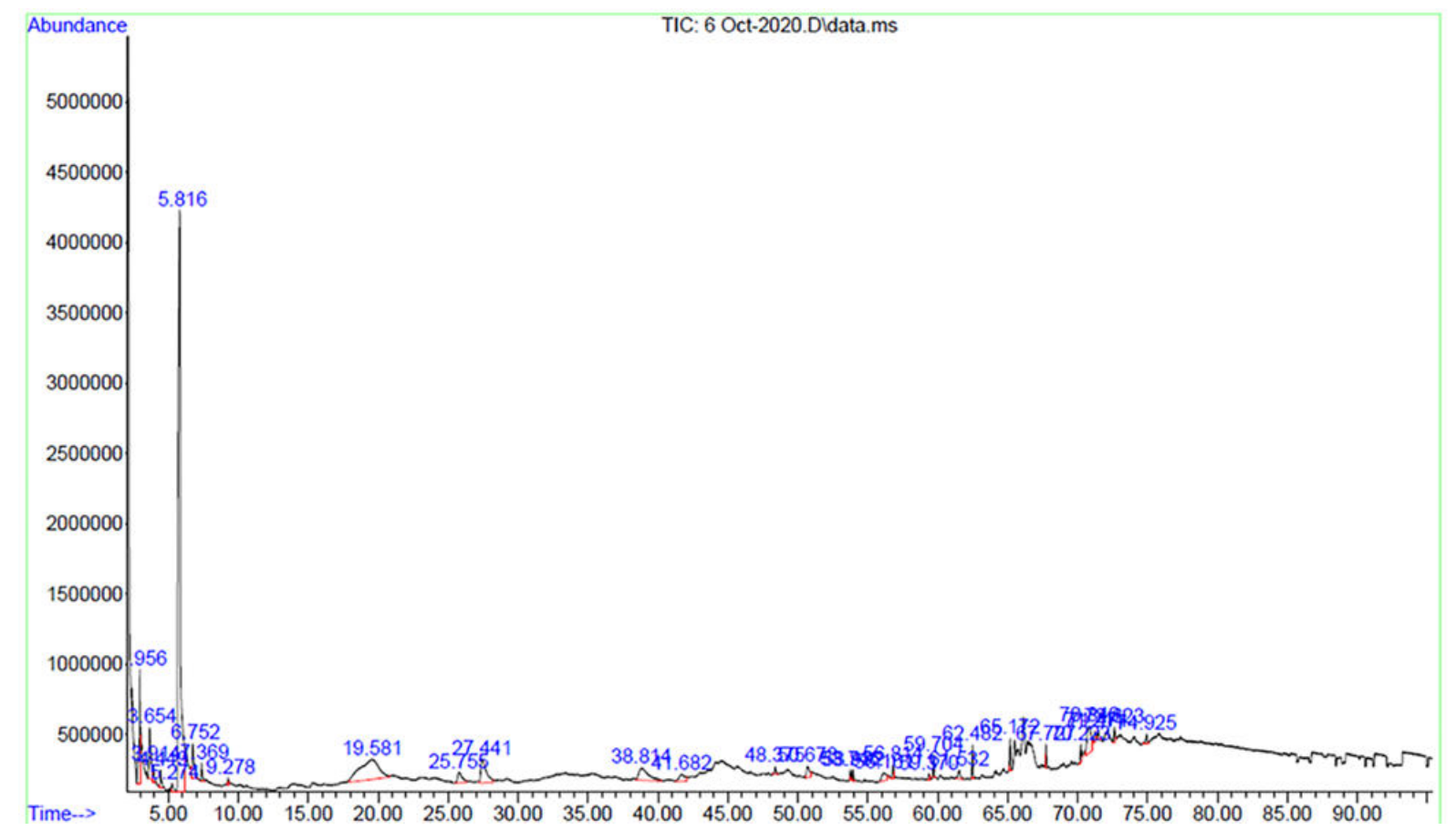

Figure 2: GC-MS chromatogram of $E$ coracana bran phenol extract.

\begin{tabular}{ccllcc}
\hline Sl.No & $\begin{array}{c}\text { RT } \\
(\text { min) }\end{array}$ & \multicolumn{1}{c}{ Compounds } & $\begin{array}{c}\text { Mol } \\
\text { Wt }\end{array}$ & $\begin{array}{c}\text { Probability } \\
(\boldsymbol{\%})\end{array}$ & $\begin{array}{c}\text { Area } \\
(\boldsymbol{\%})\end{array}$ \\
\hline 1. & 2.956 & Ammonium acetate & 77 & 34.3 & 4.568 \\
2. & 3.654 & N,N-Dimethylaminoethanol & 89 & 61.0 & 2.889 \\
3. & 3.914 & 2-Butanol, 1-(dimethylamino) & 117 & 42.8 & 0.642 \\
4. & 4.449 & Pyruvic acid, methyl ester & 102 & 70.6 & 0.637 \\
5. & 5.274 & 2-Cyclopenten-1-one & 82 & 20.2 & 0.524 \\
6. & 5.816 & Dimethylsulfoxide-D6 & 84 & 98.4 & 45.49 \\
7. & 6.752 & 2-Methyl-8-nitroisoxazolizidine & 174 & 10.2 & 1.764 \\
8. & 7.369 & Dimethylaminoethanol acetate & 131 & 51.1 & 0.645 \\
9. & 9.278 & Dimethyl trisulfide & 126 & 95.1 & 0.208 \\
10. & 19.581 & Glycerin & 92 & 82.4 & 12.134 \\
11. & 25.755 & p-Vinylguaiacol & 150 & 63.5 & 1.544 \\
12. & 27.441 & Syringol & 154 & 70.4 & 4.070 \\
13. & 38.814 & Nonanedioic acid, monomethyl ester & 204 & 57.9 & 3.734 \\
14. & 48.375 & Hexadecanoic acid, methyl ester & 270 & 25.3 & 0.329 \\
15. & 50.678 & n-Hexadecanoic acid & 256 & 66.1 & 1.105 \\
16. & 53.746 & 8,11-Octadecadienoic acid, methyl ester & 294 & 10.2 & 0.345 \\
17. & 53.932 & 11-Octadecenoic acid, methyl ester & 296 & 12.7 & 0.367 \\
18. & 56.161 & 17-Octadecynoic acid & 280 & 11.8 & 1.134 \\
19. & 56.814 & Heptadecane, 9-hexyl- & 324 & 7.28 & 0.488 \\
20. & 59.370 & Chimilether & 344 & 7.39 & 0.228 \\
21. & 59.704 & 1-Iodo-2-methylundecane & 296 & 4.02 & 0.650 \\
22. & 61.532 & 9-Octadecenoic acid, (2-phenyl-1,3-dioxolan-4-yl)methyl & 444 & 23.1 & 0.645 \\
23. & 62.482 & 1-Iodo-2-methylundecane & 296 & 4.59 & 1.035 \\
\hline & & & & & \\
\hline
\end{tabular}




\begin{tabular}{cclccc}
\hline 24. & 65.172 & Heptadecane, 9-hexyl- & 324 & 7.51 & 0.978 \\
25. & 65.484 & $\begin{array}{l}\text { 2-[4-methyl-6-(2,6,6-trimethylcyclohex-1-enyl)hexa-1,3,5- } \\
\text { trienyl]cyclohex-1-en-1-carboxaldehyde }\end{array}$ & 324 & 34.6 & 1.761 \\
26. & 66.115 & 13,17-Seco-5 $\alpha$-pregn-13(18)-en-20-one & 302 & 16.9 & 2.485 \\
27. & 66.405 & 1-Heptatriacotanol & 536 & 16.6 & 3.849 \\
28. & 67.727 & Heptadecane, 9-hexyl- & 324 & 3.75 & 0.611 \\
29. & 70.840 & B-Monoolein & 356 & 16.8 & 3.366 \\
30. & 72.623 & Octadecane, 3-ethyl-5-(2-ethylbutyl)- & 366 & 22.7 & 0.651 \\
31. & 74.925 & 1-Hentetracontanol & 592 & 46 & 0.453 \\
\hline
\end{tabular}

Table 1: GC-MS chemical composition analysis of $E$ coracana bran phenol extract.

a

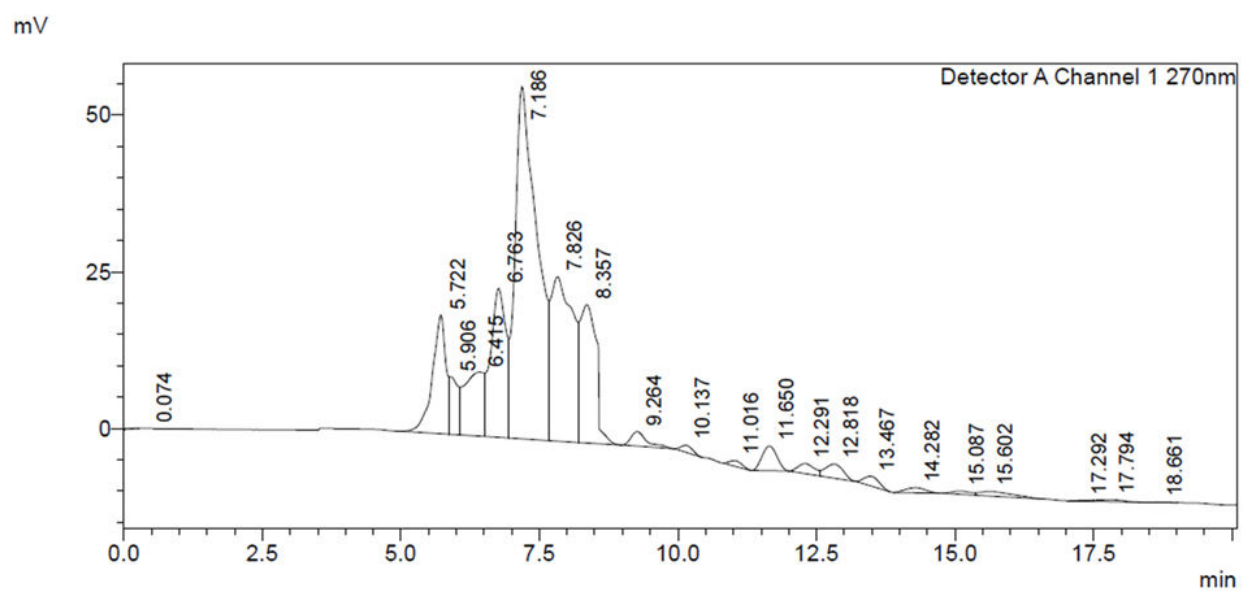

b

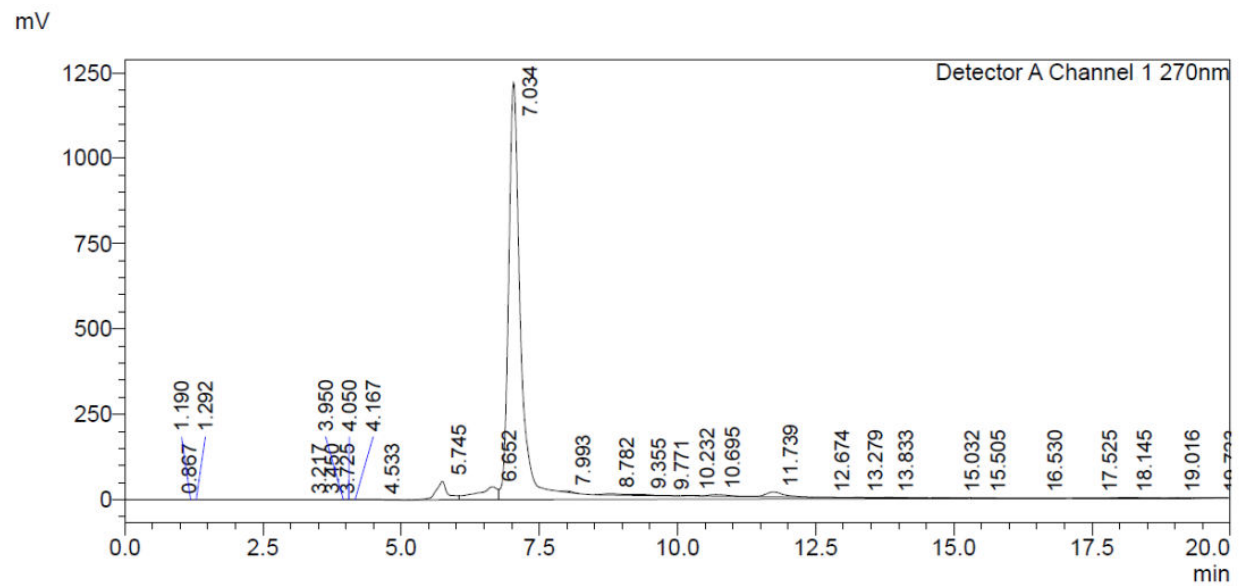

Figure 3. RP-HPLC analysis. (a) E coracana bran phenol extract and (b) syringol isolated from E coracana bran phenol extract. The compounds for HPLC were fractionated using mobile phase containing methanol: acetic acid: water (80:2:18; v/v/v) with $0.5 \mathrm{ml} / \mathrm{min}$ flow rate and absorbance was recorded at $270 \mathrm{~nm}$. 


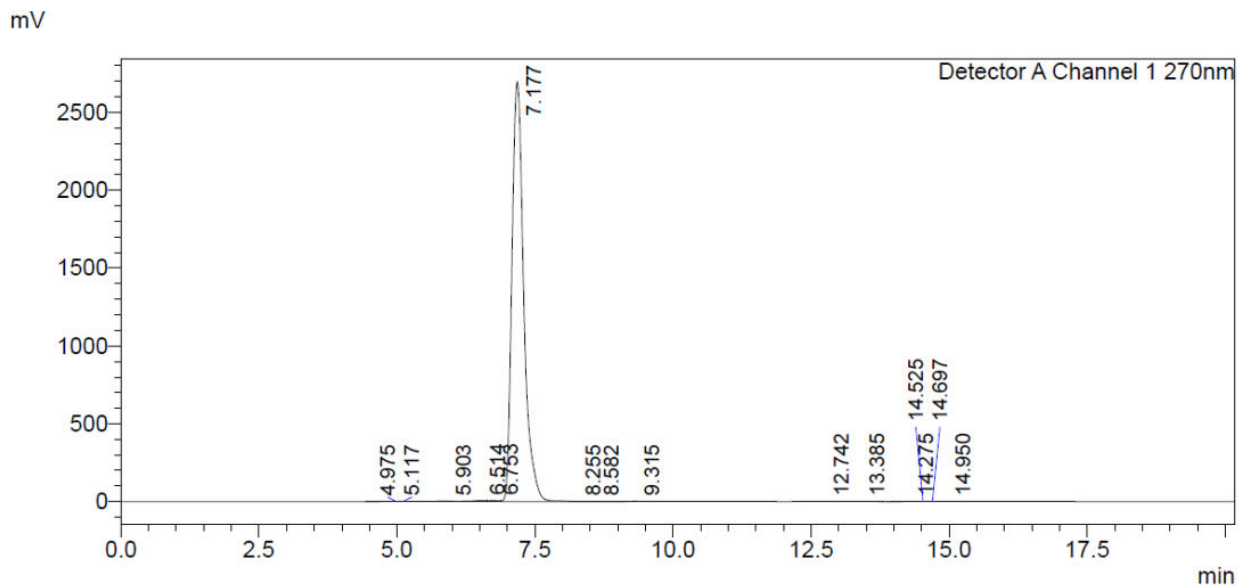

Supplementary Fig 2. RP-HPLC of standard syringol.

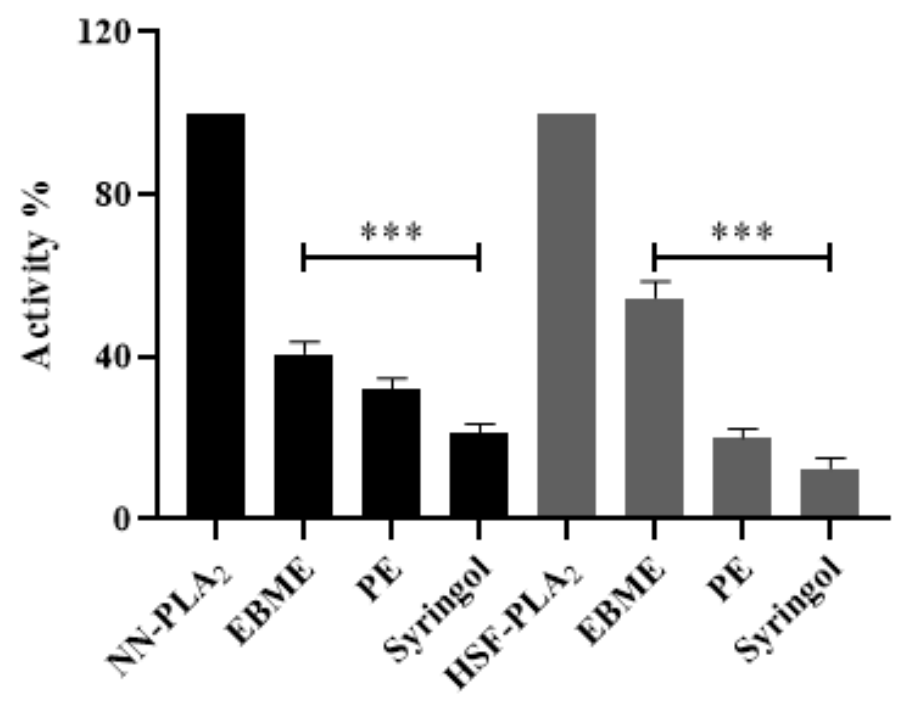

Supplementary Fig 3. Inhibition of sPLA 2 enzyme activity by EBME, PE and syringol isolated from Ecb.

\subsection{Effect of substrate and calcium concentration on inhibition of SPLA $_{2}$ enzyme activity by syringol.}

Syringol exhibited potent in vivo anti-inflammatory activity by inhibiting the $\mathrm{sLA}_{2}$ enzyme activity (Fig 4a). The inhibition was dose dependent and the extent of average inhibitory was found to be $\sim 90 \%$ (ranging between 85 to $95 \%$ ). Findings from inhibition studies indicate syringol effectively inhibits sPLA to $_{2}$ variable and yet comparable efficacies. The $\mathrm{IC}_{50}$ concentration against $\mathrm{sPLA}_{2} \mathrm{~s}$ from $N$. naja, D. russelii, HSF, and HPF was found to be 5.66, 5.20, 4.99 and $5.45 \mu \mathrm{g}$ respectively (Table 02).

The few reported inhibitors have been shown to reduce $\mathrm{SPLA}_{2}$ activity by interfering with concentration of substrate or by chelating metal ion (Davidson, Dennis et al. 1987, Francis, Seebart et al. 1992). To analyse the mechanism of $\mathrm{SPLA}_{2}$ inhibition by syringol, we carried out inhibition studies as a function of substrate 
concentration. The $\mathrm{SPLA}_{2}$ assay was carried out in the absence and presence of syringol at their given $\mathrm{IC}_{50}$ concentrations $(3 \mu \mathrm{g})$, interestingly the $\mathrm{sPLA}_{2}$ activity increases almost linearly with increased substrate concentration. The maximum inhibition by syringol was observed with substrate concentration of $90 \mathrm{mM}$. The percentage of inhibition by syringol remains constant $(\sim 50 \%)$ over entire substrate concentration range, hence the inhibition is independent of substrate concentration (Fig. 4b). Similarly, we tested the effect of calcium ions on inhibitory activity of syringol at $\mathrm{IC}_{50}$ concentration $(3 \mu \mathrm{g})$. An increased calcium concentration from 0 to 25 $\mathrm{mM}$, increases $\mathrm{SPLA}_{2}$ activity linearly but did not change the extent of inhibition ( $\left.50 \%\right)$ (Fig. 4c). This data indicates that sPLA 2 inhibition by syringol is independent of substrate as well as calcium concentration.
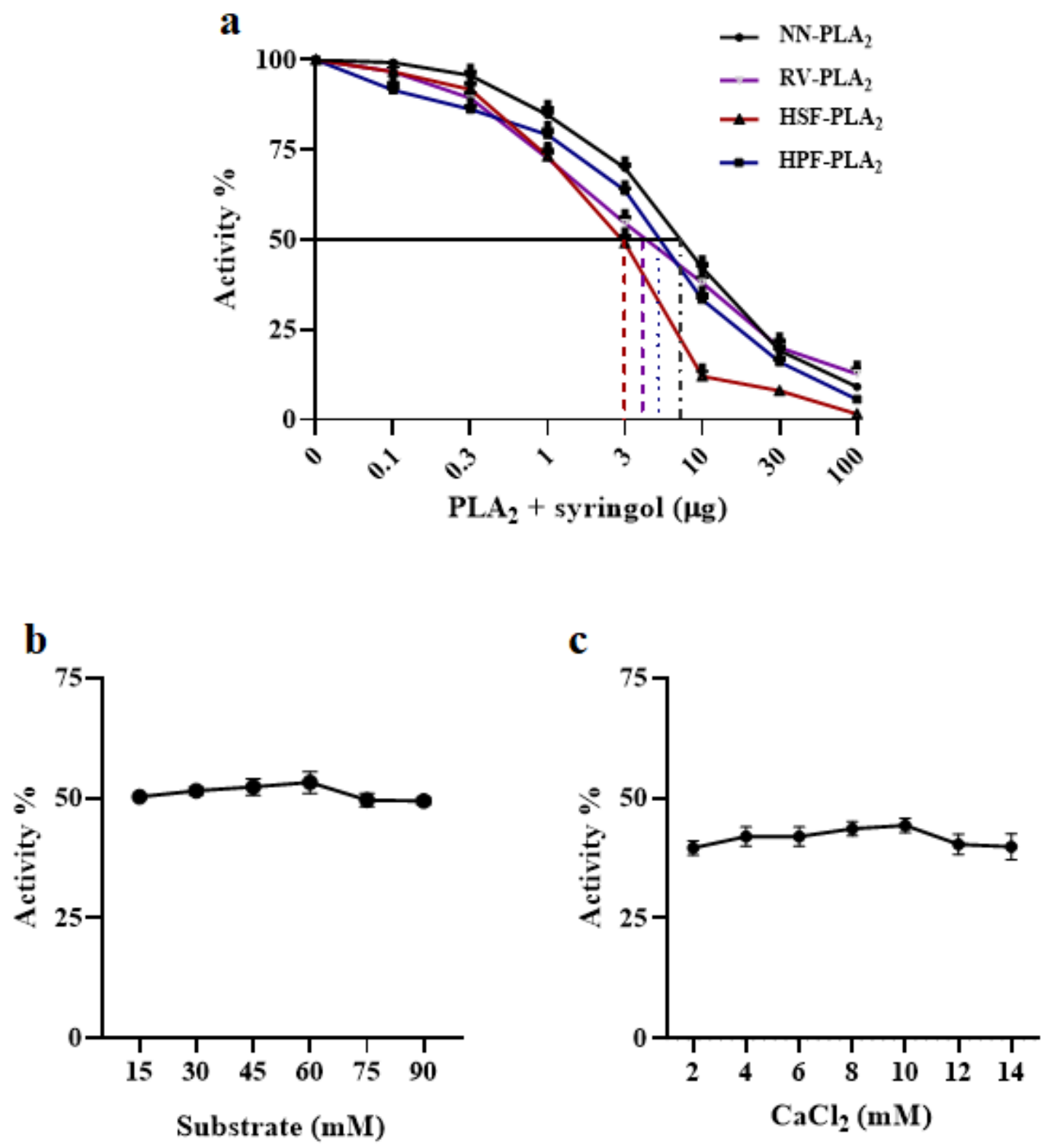
Fig. 4. Inhibition of $\mathrm{SPLA}_{2}$ and effect of substrate and calcium chloride concentration on $\mathrm{PLA} \mathrm{A}_{2}$ inhibition:

(a) Different concentration of syringol was pre-incubated with $30 \mu \mathrm{g}$ of $\mathrm{sPLA}_{2}$, adding to a final volume of 260

$\mu 1$. The mixture was incubated for $40 \mathrm{~min}$ at $37^{\circ} \mathrm{C}$, its absorbance was recorded at $425 \mathrm{~nm} 10 \mathrm{~min}$ intervals. (b) Percent activity of $\mathrm{sPLA}_{2}$ with $\mathrm{IC}_{50}$ concentration syringol with varying concentration of substrate and (c) calcium chloride. Data represents Mean $\pm \operatorname{SD}(n=3)$.

\begin{tabular}{|l|c|}
\hline \multirow{2}{*}{ Source of sPLA } & IC $_{\mathbf{5 0}}$ \\
\cline { 2 - 2 } & Syringol $(\boldsymbol{\mu g})$ \\
\hline N. naja PLA $_{2}$ & 7.50 \\
\hline D. russelii PLA $_{2}$ & 5.00 \\
\hline HSF PLA & \\
\hline HPF PLA $_{2}$ & 3.00 \\
\hline
\end{tabular}

Table 2: Determination of $\mathbf{I C}_{\mathbf{5 0}}$ values: $\mathrm{IC}_{50}$ values represent the concentration of syringol that inhibits the $\mathrm{sPLA}_{2}$ activity by $50 \%$.

3.5 Neutralization of $\mathrm{SPLA}_{2}$ induced mice paw edema via downregulation of the expression of proinflammatory signaling mediators expression by syringol.

Potent in vitro $\mathrm{sPLA}_{2}$ inhibitory activity of syringol in vitro encouraged us to evaluate its effect on edema inducing activity. Mice hind paw was injected with $4 \mu \mathrm{g}$ of $\mathrm{sPLA}_{2}$ to induce the inflammatory reaction and resulted in swelling of footpad with an edema ratio of $173.50 \pm 7.8 \%$. Group of animals injected with syringol reduced the edema in concentration dependent manner. $3 \mu \mathrm{g}$ of syringol significantly reduced edema ratio to $108.5 \pm 2.1 \%$ with reduced swelling of foot pad (Fig. 5a).

Recently, Toyama et al. reported that edema induced by SPLA $_{2}$ results in activation of cytosolic PLA 2 $\left(\mathrm{CPLA}_{2}\right)$ (Toyama, Costa et al. 2019) and release of arachidonic acid (AA) from membrane phospholipids. Released AA by both SPLA $_{2}$ and $\mathrm{cPLA}_{2}$ is further activates COX-2 and oxidized to produce various proinflammatory signaling mediators such as p-cPLA $2, \mathrm{p}-\mathrm{COX}-2, \mathrm{p}-\mathrm{IkB} \alpha, \mathrm{p}-\mathrm{p} 38$ and MPO (Monteseirín, Bonilla et al. 2001, Schieven 2005, Arlier, Kayisli et al. 2018). Edematous tissues from control and treatment groups were examined for the expression levels of pro-inflammatory signaling mediators. Interestingly, the syringol decreases the phosphorylation of pro-inflammatory signaling mediators in a dose dependent manner $(0.5,1.0 \&$ 
$3.0 \mu \mathrm{g}$ ) as shown in Fig. 5b. The decrease in swelling and footpad thickness was observed in syringol treated group of mice compare to $\mathrm{sPLA}_{2}$ treated mice (Fig c \& d). To examine the effects of syringol on the histopathological changes in $\mathrm{SPA}_{2}$ induced paw edema, sections of paw tissue from experimental groups were stained by H\&E. A large number of infiltrating inflammatory cells were observed in the paw tissue after sPLA 2 injection compared with the control group. Syringol at a dose of $3 \mu \mathrm{g}$ resulted in decreased number of inflammatory cell infiltration (Fig. 5e). This data correlates with decreased level of MPO phosphorylation. Similarly, reduced level of interstitial fluid was also observed in syringol treated group compared to SPLA $_{2}$ induced edematous tissue. These findings highlight the involvement of syringol in regulation of inflammatory responses in vivo.
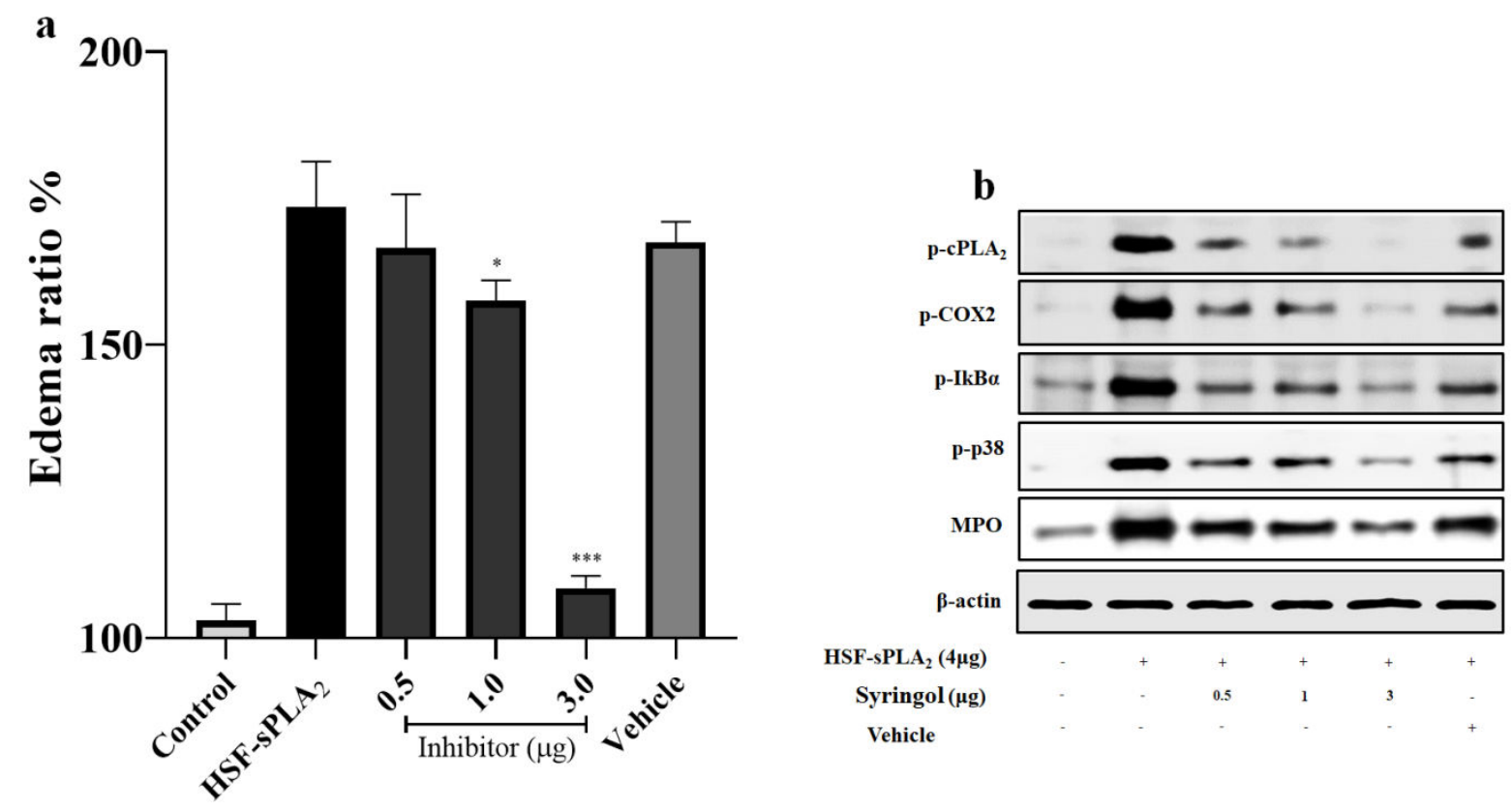

C

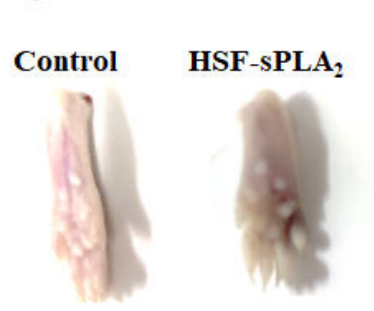

\section{$0.5 \mu \mathrm{g}$} Syringol

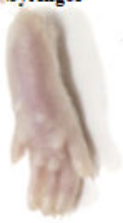

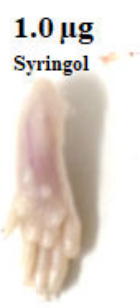

d

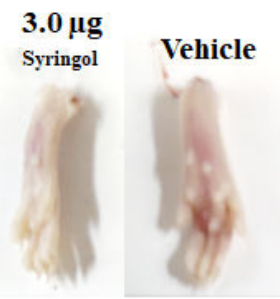

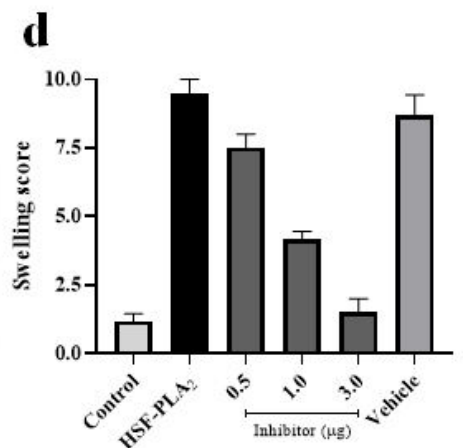




\section{$\mathbf{e}$}

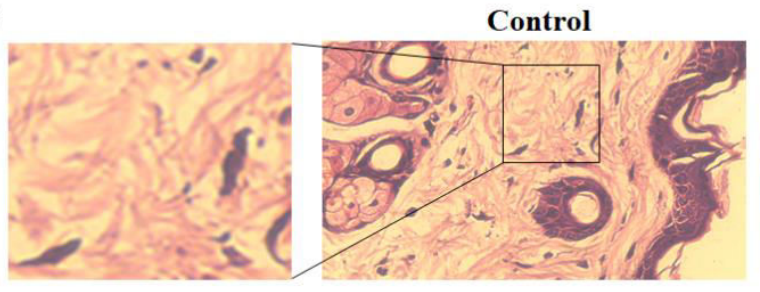

$\mathrm{sPLA}_{2}+0.5 \mu \mathrm{g}$ Syringol

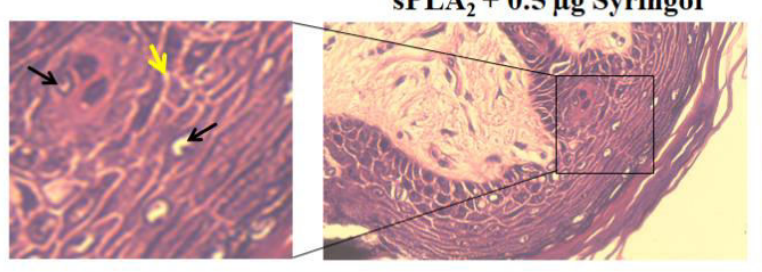

$\mathrm{sPLA}_{2}+3 \mu \mathrm{g}$ Syringol

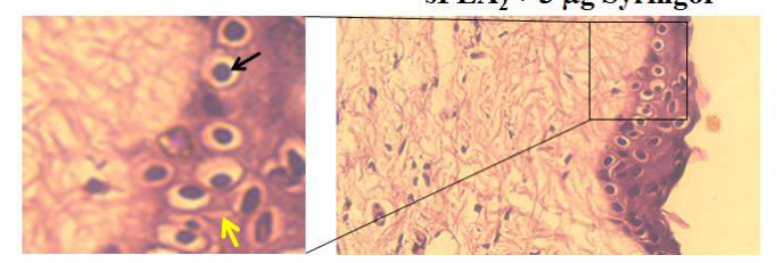

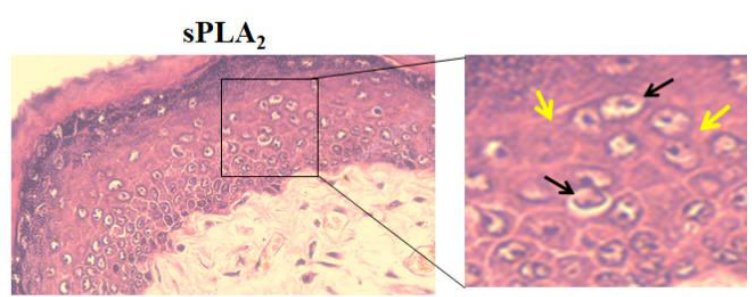

$\mathrm{sPLA}_{2}+1 \mu \mathrm{g}$ Syringol

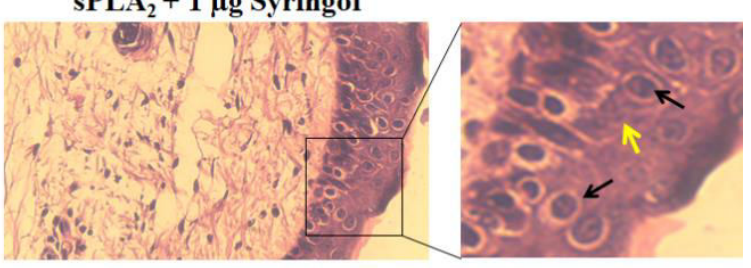

$\mathbf{s P L A}_{2}+$ Vehicle

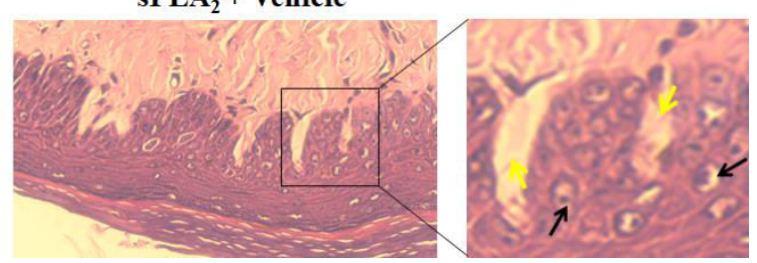

Fig. 5. Neutralization of edema by syringol. (a) Mice paw edema was induced by $4 \mu \mathrm{g}$ of $\mathrm{sPLA}_{2}$ with or without different dose of syringol in a total reaction volume of $40 \mu \mathrm{l}$. All animals were scarified after $45 \mathrm{~min}$ and dissect the footpad to observe edema. (b) Footpad tissue lysates from treatment and control groups were analyzed for the activation of $\mathrm{cPLA}_{2}, \mathrm{COX}-2, \mathrm{IkB}-\alpha, \mathrm{p} 38$ and MPO in mice footpad lysate by western blots. $\beta-$ actin was used as a loading control. (c) Edematous footpad of mice. (d) Visual scoring of footpad thickness. (e) Different groups of mice were treated with $\mathrm{SPLA}_{2}$, syringol/vehicle were sacrificed and paw tissues were processed for histological staining. Red arrow indicates infiltration of cells and black arrow indicates deposition of edema fluid. ${ }^{*} \mathrm{p}<0.05$ and ${ }^{* * *} \mathrm{p}<0.001$ as compared to $\mathrm{sPLA}_{2}$ alone.

\subsection{Effect of syringol on 5-LOX activity}

5-LOX inhibitory potential of syringol was evaluated by spectrophotometrical measurement of the formation of 5-HETE. The syringol completely inhibited the 5-LOX activity at the dose of $2.5 \mu \mathrm{g}$ and the inhibition is concentration dependent manner with an $\mathrm{IC}_{50}$ value of $500 \mathrm{ng}$ (Fig. 6). Nordihydroguaiaretic acid (NDGA), a known, potent inhibitor of 5-LOX enzyme was used as a positive control. 


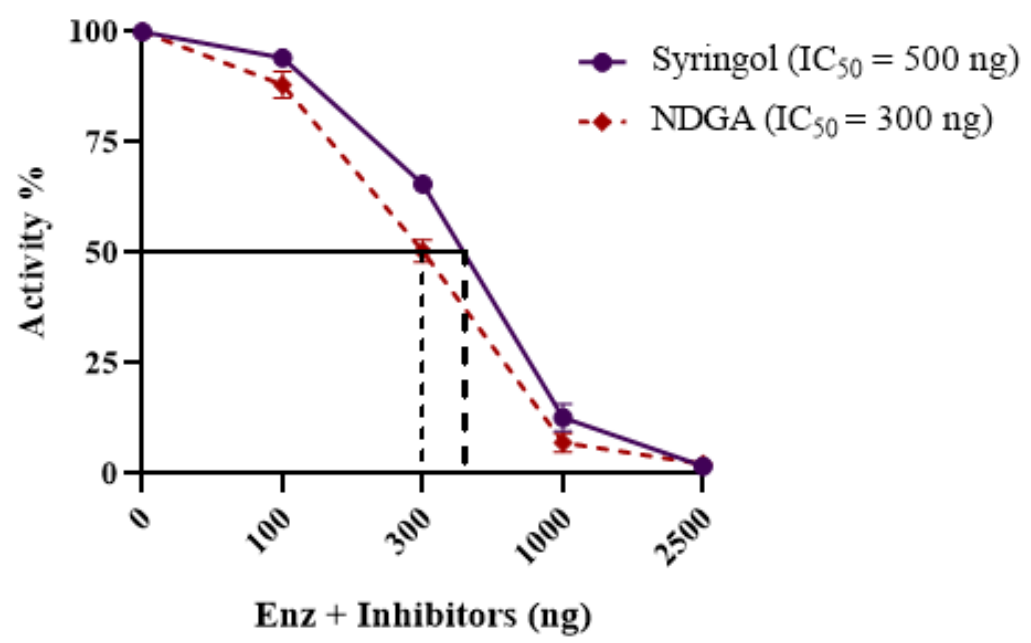

Fig. 6. Inhibition of human 5-LOX by syringol: Human 5-LOX was incubated with different doses of syringol. The reaction mixture contained $100 \mathrm{mM}$ phosphate buffer $\mathrm{pH}$ 7.4, $50 \mathrm{mM}$ DTT , $200 \mathrm{mM} \mathrm{ATP,} 300 \mathrm{mM} \mathrm{CaCl}_{2}$ and $5 \mu \mathrm{g}$ of enzyme pre-incubated with syringol for 3 min separately. Reaction was initiated by adding $150 \mathrm{mM}$ AA. The molar extinction coefficient of $25,000 / \mathrm{M} / \mathrm{cm}$ was used to calculate specific activity of the enzyme. NDGA was used as a positive control. Data represents mean $\pm \operatorname{SD}(n=3)$.

\subsection{Interaction studies of syringol against anti-inflammatory enzymes}

Results from initial experiments indicate that syringol inhibits $\mathrm{SPLA}_{2}$ enzyme activity by direct interaction. To confirm these results, we determined intrinsic fluorescence measurements and also performed the $\mathrm{SPLA}_{2}$ activity following dialysis. Intrinsic fluorescence is used to demonstrate the interaction of compound with enzyme, alteration in fluorescence reflects the conformational change in enzyme due to direct interaction of substrate or ligand with enzyme. As shown in the (Fig. 7a) fluorescence quenching was observed with increasing in the concentration (0 to $1000 \mathrm{ng}$ ) of syringol. Intrinsic fluorescence spectra of $\mathrm{SPLA}_{2}$ were measured in the presence and absence of syringol. Another experimental method to demonstrate the direct interaction of enzyme-inhibitor complex is by dialysis. $\mathrm{SPLA}_{2}$ activity remained unaltered even after extensive dialysis indicating the direct interaction of inhibitor with enzyme. On extensive dialysis of sPLA 2 and syringol, the mixture retained the same inhibitory activity (Fig. 7b). These data demonstrate that syringol may interact directly with $\mathrm{SPLA}_{2}$ to inhibit its activity.

3D structures of syringol and crystal structures of anti-inflammatory targets ( $\mathrm{sLA}_{2}$, and COX-2) were prepared as described in methods section and were analysed by IFD method. For docking studies crystal structure of anti-inflammatory targets were downloaded from Protein Data Bank server. Syringol forms 
hydrogen bond (H Bond) with N. naja sPLA $_{2}\left(\mathrm{NN}-\mathrm{sPLA}_{2}\right)$ active site amino acid Cys 44. In contrast, syringol forms $\mathrm{H}$ bond with His 27, Gly 29, Gly 31 and Asp 48, the active site residues of HIE-sPLA 2 and also formed $\mathrm{H}$ bond with the cofactor calcium ion. On the other hand, syringol interacted with active site amino acid residue Lys 69 of $D$ russelii sPLA $_{2}\left(\mathrm{DR}-\mathrm{SPLA}_{2}\right)$ and did not interact with the cofactor of the enzyme. When syringol was subjected to IFD with human COX-1, it interacts only with a Ser 504 at the active site. Syringol forms two $\mathrm{H}$ bond with Arg 120 at the active site of COX-2 enzyme. H bond was formed with Tyr 181 between syringol and active site of 5-LOX (Fig. 7c [i to vi]). The glide score and energy values of syringol with antiinflammatory targets were compared (Fig. 7c [vii \& viii]). The computational docking studies (in silico) revealed that syringol is a weak binder.

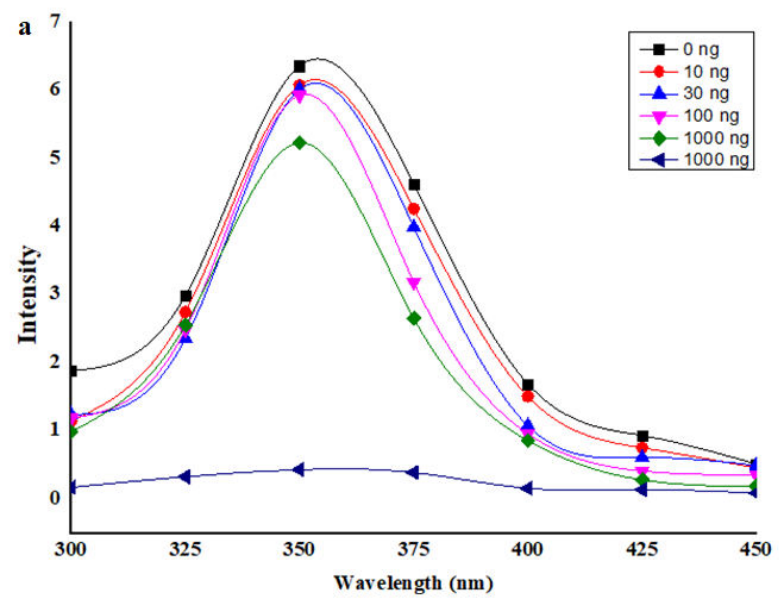

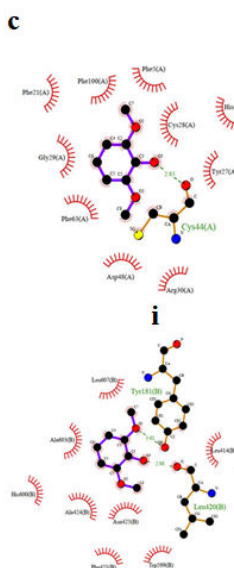

iv

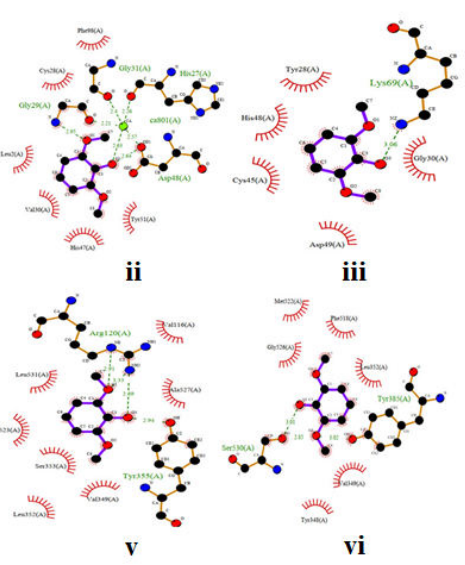

viii

\begin{tabular}{|c|c|c|}
\hline \multirow{2}{*}{$s \mathrm{PLA}_{2}+\mathrm{BrE}$} & \multicolumn{2}{|c|}{ Inhibition (\%) } \\
\hline & Before dialysis & After dialysis \\
\hline Nnaja $\mathrm{PLA}_{2}+$ syringol & 48.50 & 52.35 \\
\hline D russellii $\mathrm{PLA}_{2}+$ syringol & 49.00 & 49.55 \\
\hline HSF PLA ${ }_{2}+$ syringol & 51.71 & 54.33 \\
\hline HPF PLA $A_{2}+$ syringol & 46.25 & 52.46 \\
\hline
\end{tabular}
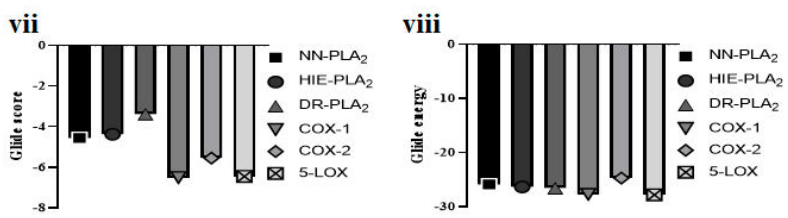

\begin{tabular}{|c|c|c|c|}
\hline $\begin{array}{l}\text { Sl. } \\
\text { No }\end{array}$ & Enzyme & \begin{tabular}{|c|}
$\begin{array}{c}\text { Docking score } \\
(\mathrm{kcal} / \mathrm{mol})\end{array}$ \\
\end{tabular} & $\begin{array}{c}\text { Glide energy } \\
\text { (kcal/mol) }\end{array}$ \\
\hline a. & $\mathrm{PLA}_{2}$ (Naja naja) & -4.56 & -25.86 \\
\hline a. & $\begin{array}{l}\text { PLA }_{2} \text { (Human inflammatory } \\
\text { exudate) }\end{array}$ & -4.37 & -26.30 \\
\hline a. & $\mathrm{PLA}_{2}$ (Daboia russelii) & -3.39 & -26.57 \\
\hline a. & COX-1 & -6.50 & -27.69 \\
\hline a. & $\mathrm{COX}-2$ & -5.54 & -24.69 \\
\hline a. & 5-LOX & -6.44 & -27.79 \\
\hline
\end{tabular}

Fig. 7. Interaction studies of syringol and $\mathbf{S P L A}_{2}$. (A) Intrinsic fluorescence spectra of $100 \mu \mathrm{g} \mathrm{sPA}_{2}$ was measured in the absence and presence of different concentrations of syringol. The reaction mixture contained Tris- $\mathrm{HCl}$ buffer ( $\mathrm{pH} 7.4)$ and $5 \mathrm{mM}$ calcium. The solutions were excited at $280 \mathrm{~nm}$ and emission was recorded at 300 to $450 \mathrm{~nm}$. (B) Inhibition of PLA $\mathrm{P}_{2}$ enzyme activities by syringol before and after dialysis. (C) Energetically favourable binding modes of syringol calculated using induced fit molecular docking method. The hydrogen 
bonding and hydrophobic interactions between the syringol and $\mathrm{SPLA}_{2}$ from different source (i to vi) are depicted using the LigPlot software. (vii) glide score and (viii) glide energy (calculated in kcal/mol) associated with best binding modes of syringol with the active site of various inflammatory enzymes. (D) Glide score and glide energy values of syringol with $\mathrm{SPLA}_{2}$.

Overall, these studies suggest that the purified molecule syringol from Ecb inhibits pro-inflammatory enzymes ( $\mathrm{SLA}_{2}, 5-\mathrm{LOX}$ and COX-2) in vitro. Mouse paw edema was alleviated by syringol and it was shown to modulate the activity of pro-inflammatory $\mathrm{CPLA}_{2}, \mathrm{COX}-2, \mathrm{I} \kappa \mathrm{B} \alpha$ and MPO.

\section{Discussion:}

Inflammation is complex of biological reaction in response to injury, infection, allergy, irritation, noxious agent and cell damage. It is clinically characterized by redness, warm, swelling, and pain due to infiltration of immune cells at the site of inflammation (Mourits, Prummel et al. 1997). Inflammation is associated with many clinical conditions such as rheumatoid arthritis, cardiovascular diseases, cancer, allergy, asthma, inflammatory bowel disease, diabetes, Parkinson's disease, skin conditions like eczema, acne, and psoriasis. These conditions affect millions of people across the globe. Therefore, management and treatment of inflammation is critical to maintain the good health and quality lifestyle. The available medicines to treat inflammatory diseases include non-steroidal anti-inflammatory drugs (NSAIDs), corticosteroids, immunosuppressants and other synthetic drugs. Prolonged usage of these drugs will possess serious and harmful side effects on human health. Therefore, development of alternative treatment would be a better strategy in order to overcome the adverse effects and to identify an effective drug to treat inflammation.

One of the important alternative sources of therapeutic drugs is plant based natural products, especially phytochemicals that produce pharmacological effects on human health (Dillard and German 2000). In recent years, understanding the role of phytochemicals in regulation of inflammation has grown substantially. Among all phytochemicals, polyphenols are considered as the major anti-inflammatory constituents from sources such as fruits, vegetables, tea, grains, and legumes (Larrosa, Yañéz-Gascón et al. 2009, Krzyzanowska, Czubacka et al. 2010). The collective evidences suggest that these polyphenols are involved in a variety of reactions as a consequence of inflammation with an anti-inflammatory activity through the inhibition of arachidonic aciddependent enzymatic pathways such as $\mathrm{SPLA}_{2}, \mathrm{COX}$ and LOX which are activated in the course of the inflammatory process (Yahfoufi, Alsadi et al. 2018). However, to the best of our knowledge very few have been attempted in clinical studies (Rasouli, Farzaei et al. 2017). Our study aimed at the isolation, bio-active guided 
purification and characterization of anti-inflammatory molecule from the E. coracana bran (Ecb) and whole seed. Among the extractions, E coracan bran methanol extract (EBME) shows significant pro-inflammatory $\mathrm{SPLA}_{2}$ inhibition in dose dependent manner both in vivo and in vitro, indicating the presence of antiinflammatory molecule. Hence further purification and characterization studies were performed using this fraction. Purification of syringol from EBME was carried out by the aid of biophysical techniques such as GCMS and RP-HPLC.

Syringol (2,6-dimethoxyphenol) and its related compounds are combustion products of lignins, phenolic polymers found primarily in hardwood. They are major constituents of wood smoke condensate and identified as having a woody/herby flavor and smoky odor (Maga 1987). Syringol is also been isolated from rice straw derived bio-oil (Hao, Chen et al. 2016). Apart from being a constituent of wood smoke, syringol is shown to exhibit different bio-functions. Loo et. al, (Loo, Jain et al. 2008) isolated syringol from the pyroligneous acid, Rhizophora apiculate which is shown to have antioxidant activities and a study conducted by the Azeem Intisar et. al, reported the anti-cancer nature of syringol on human hepatocellular carcinoma cell lines (HCCLM3) (Intisar, Zhang et al. 2012). US patent filed by Sung-Ho Wang claimed several pharmaceutical properties of syringol such as antioxidant, blood glucose level regulator, hangover relaxant and a molecule to treat atopic dermatitis (Wang 2007). Likewise, several phyto-phenolics are reported as effective molecules with antiinflammatory properties (Chen, Chen et al. 2018, Li, Zhang et al. 2019, Yin, Wu et al. 2019, Zhu, Gu et al. 2019). Our experiments demonstrated that syringol comprises anti-inflammatory activity by inhibiting sPLA $_{2}$ enzyme. Hence further work is designed with a deliberation that the purified molecule is syringol. To get insight into the $\mathrm{SPLA}_{2}$ inhibitory properties of isolated molecule, we conducted both in vitro and in vivo studies.

As demonstrated by Davidson et al, their inhibition was relieved by increasing the substrate concentration (Davidson, Dennis et al. 1987). Conversely, in this study we found that inhibition of SPLA $_{2}$ activity by syringol is not relieved by increased substrate concentration. Thus, the inhibition is independent of substrate concentration. Brian Francis et al. proved that citrate inhibits Bothrops asper sPLA 2 enzyme by chelating calcium ion (Francis, Seebart et al. 1992). Calcium is a co-factor of sPLA 2 enzyme, some of the sPLA 2 inhibitors appear to be calcium dependent and their inhibitory mechanism is mediated by displacement of catalytically essential calcium from the enzyme (Pruzanski, Greenwald et al. 1992). To exclude the possibility of metal chelation we demonstrated that increasing the calcium concentration does not relieve the sPLA $_{2}$ inhibition by syringol, which is in accordance with our previous studies (Jameel, Shekhar et al. 2006). From 
these experimental evidences we can conclude that inhibition of $\mathrm{SPLA}_{2}$ by syringol is independent of calcium ion concentration and substrate concentration.

In addition to in vitro anti-inflammatory activity we also evaluated the in vivo anti-inflammatory activity of syringol by using well established mouse paw edema model. Edema referred as swelling due to excessive accumulation of fluid in the tissue is strongly associated with prostaglandin production, COX-2 upregulation and the formation of reactive free radicals as well as other inflammatory mediators (Sharma, Westman et al. 1994). Inflammatory $\mathrm{SPLA}_{2} \mathrm{~s}$ from snake venoms and synovial fluid on injection into the animal foot paw induce acute inflammatory response with edema, swelling of synovial cells and hyperplasia (Elford, Graeber et al. 1992). Co-injection of sPLA 2 enzyme with inhibitors reduces the edema and pro-inflammatory mediators as shown by several studies (Joshi, Venkatesha et al. 2016). A study conducted by Vishwanath et al. showed that Aristolochic acid, a sPLA 2 inhibitor reduces the swelling and redness of mice footpad and thus inhibited the edema (Vishwanath, Fawzy et al. 1988). Amy Burdette et al. showed that the phenolic compound isolated from S. bicolor reduced the TPA - induced ear edema by reducing myeloperoxidase (MPO) activity in mice model (Burdette, Garner et al. 2010). Syringol with different concentrations was co-injected along with $\mathrm{sPLA}_{2}$ enzyme. The experimental results show a significant reduction of edema ratio in the group of animals injected with syringol indicating its certain anti-inflammatory properties.

To substantiate the anti-inflammatory effect and to understand the molecular mechanism behind the syringol induced anti-inflammatory effect, we examined its effect on expression of other pro-inflammatory signaling mediators. During edema cPLA 2 is rapidly activated by increased level of intracellular calcium (Clark, Schievella et al. 1995) and get phosphorylated at Ser 505 by mitogen-activated protein kinase (MAPK) (Gijón and Leslie 1999). Activated cPLA $_{2}$ hydrolyzed the membrane phospholipids, as a result the AA was released and oxidized by COX-2 to produce pro-inflammatory prostaglandins (Suram, Gangelhoff et al. 2010). Furthermore, tissue lysates from mice paw treated with $\mathrm{SPLA}_{2}$, syringol was checked for the phosphorylation of $\mathrm{cPLA}_{2}, \mathrm{p} 38$ and COX-2 enzymes. Interestingly syringol reduced the phosphorylation of cPLA 2 , p83 and COX2 , thus inhibited the expression levels of pro-inflammatory signaling mediators. Abnormal or excessive stimulation of $N F-\kappa B$ is observed in inflammation and NF- $\kappa B$ protein activation plays a key role in the progression of inflammation by inducing the expression of pro-inflammatory genes. In normal conditions NF$\mathrm{kB}$ is associated with $\operatorname{IkB} \alpha$ and during inflammation the cytokines and other pro-inflammatory mediators phosphorylates the IkB $\alpha$ and results in the activation and release of NF-kB (Conner and Grisham 1996). Hence inhibiting the activation of NF-kB by blocking the phosphorylation of $\mathrm{IkB} \alpha$ is crucial. Kyung-Min Lee et al. 
reported that activation of NF-kB results in up regulation of COX-2 expression. Activation of NF-kB leads to transcription of pro-inflammatory cytokines, inflammatory enzymes and inducible nitric oxide synthase (Lawrence 2009). The experimental results show that syringol could inhibit the expression of NF- $\kappa \mathrm{B}$ signalling pathway by inhibiting the phosphorylation levels of $\mathrm{I} \kappa \mathrm{B} \alpha$, thereby playing an anti-inflammatory role, suggesting that syringol may be an $\mathrm{I} \kappa \mathrm{B} \alpha$ inhibitor. During inflammation, neutrophils are recruited and degranulated, as a result MPO was released at the site of inflammation (Bradley, Priebat et al. 1982). The MPO is a marker enzyme for neutrophils infiltration and increased many folds during inflammation, our data shows that syringol reduces the phosphorylation of MPO expression and thus decreased infiltration of neutrophils. The infiltration of inflammatory cells in edema tissue was visualised using hematoxylin and eosin stain and animals treated with syringol decreases the cells infiltration.

Furthermore, the inhibition is irreversible as demonstrated by dialysis experiment suggests that syringol may interact directly with the enzyme and forms an enzyme-syringol complex. To substantiate this further, spectrofluorometric analysis was performed. The intrinsic fluorescence emission spectra are commonly used to demonstrate interaction of molecule with protein. Direct interaction of molecules with protein will change the intrinsic fluorescence reflects conformational change in protein due to direct ligand-protein interaction. Previous studies have confirmed that some $\mathrm{sPLA}_{2}$ inhibitors change the intrinsic fluorescence spectra of $\mathrm{sLA}_{2}$ enzyme either by decreasing or increasing the intensity (Jameel, Shekhar et al. 2006). In this study altered intrinsic fluorescence intensity of $\mathrm{sPA}_{2}$ was observed in the presence of syringol suggesting that syringol interacts directly with the enzyme to form an enzyme-inhibitor complex. Few studies showed that, a weak binder can remarkably have inhibited in vitro and in vivo activity of the enzyme or their target. For instance, Wang, et al. recently proved that luteolin, a multi target kinase inhibitor showed poor interaction in molecular docking studies but luteolin inhibited the production of IL-6 and TNF- $\alpha$ by inhibiting kinases of MAPK signalling pathway (Wang, Guo et al. 2017). In same manner syringol remarkably inhibited the in vitro and in vivo activity of anti-inflammatory enzymes even though syringol had shown weak interactions in in silico methods. Collectively, this study provide the confirmation that syringol might be a promising candidate for the treatment of inflammation.

\section{Conclusion:}

Syringol, a phenolic compound was isolated and purified to homogeneity from Ecb extract. Syringol blocked the eicosanoid pathway by inhibiting $\mathrm{SPLA}_{2}, 5-\mathrm{LOX}$ and COX-2 enzyme activities, thus biosynthesis of 
pro-inflammatory mediators are reduced. The inhibition of $\mathrm{SPLA}_{2}$ is independent of substrate and calcium concentrations and irreversible. Further, syringol inhibited the paw edema induced by SPLA $_{2}$ and $\lambda$-carrageenan in vivo. The western blots carried out using edematous tissues extract demonstrated that, administration of syringol to the mice hind paw may inhibits the edema by decreasing the phosphorylation of pro-inflammatory signaling mediators. This data suggests that apart from direct $\mathrm{SPLA}_{2}$ interactions, syringol may also involve largely in regulation of inflammatory process.

\section{Declaration of competing interest:}

The authors involved in this study declare no conflict of interest.

\section{CRediT author contribution statement:}

1. Milan Gowda M D - Conceptualization, Methodology, Validation, Writing - Original Draft, Resources, Data Curation, Investigation.

2. Jayachandra K - Methodology, Validation, Investigation.

3. Vikram Joshi - Methodology, Validation, Investigation.

4. Manjuprasanna V N - Methodology, Validation, Investigation.

5. Rudresha G V - Methodology, Validation, Investigation.

6. Devadasan Velmurugan - GC-MS methodology and Validation.

7. Raman Pachaiappan - Molecular docking methodology, Validation.

8. Noor Mohamed Jameel - Conceptualization, Writing - Original Draft, Supervision.

9. Bannikuppe S. Vishwanath - Conceptualization, Writing - Original Draft, Supervision, Project administration.

\section{Funding:}

This work was supported by UGC-Major Research Project [F.No.43-41/2014 (SR)], University Grants Commission New Delhi, Government of India and DST- Promotion of University Research and Scientific Excellence (Purse) Grant (Order No.UOM/DST-PURSE/013/2015-16), Government of India, for financial assistance.

\section{Acknowledgements:}


Mr. Milan Gowda M D thank to UGC-BSR [F.No.43-41/2014 (SR)] and DST-PURSE (Order No.UOM/DSTPURSE/013/2015-16) to provide fellowships. Mr. Jayachandra K (Letter No. 09/119(0207)2019-EMR-I) thank University Grants Commission (UGC), New Delhi. Mr. Manjuprasanna V N [DV2/30/PDF/PA/IOE/201011(Vol-II) \& DST/KSTePS/Ph.D. Fellowships/CHE-01: 2018-19] and Dr. Rudresha G V [No.F41/2006(BSR)/7-366/2012(BSR) dated 22-10-2013] for fellowships. Institute of Excellence [Funded by Ministry of Human Resource Development (MHRD), Government of India], University of Mysore, Mysuru. Authors thank Central Animal Facility, University of Mysore, for providing animals. Authors thank Prof Sathish S and Mr. Chandra Mohan for Providing HPLC facility. 


\section{References:}

Arlier, S., U. A. Kayisli and A. Arici (2018). "Tumor necrosis factor alfa and interleukin 1 alfa induced phosphorylation and degradation of inhibitory kappa B alpha are regulated by estradiol in endometrial cells." Turk J Obstet Gynecol 15(1): 50-59.

Awika, J. M., L. W. Rooney, X. Wu, R. L. Prior, L. J. J. o. a. Cisneros-Zevallos and f. chemistry (2003). "Screening methods to measure antioxidant activity of sorghum (Sorghum bicolor) and sorghum products." Agric Food Chem 51(23): 6657-6662.

Bradley, P. P., D. A. Priebat, R. D. Christensen and G. Rothstein (1982). "Measurement of cutaneous inflammation: estimation of neutrophil content with an enzyme marker." J Investigative Dermatology 78(3): 206-209.

Breyer-Brandwijk, M. G. J. T. M., P. P. o. Southern, E. A. b. a. A. o. t. Medicinal, C. C. other Uses, Pharmacological Effects, T. i. Man and Animal. (1962). "The Medicinal and Poisonous Plants of Southern and Eastern Africa being an Account of their Medicinal and other Uses, Chemical Composition, Pharmacological Effects and Toxicology in Man and Animal." (Edn 2).

Budhwar, S., M. Chakraborty, K. Sethi and A. Chatterjee (2020). "Antidiabetic properties of rice and wheat bran-A review." J Food Biochem: e13424.

Burdette, A., P. L. Garner, E. P. Mayer, J. L. Hargrove, D. K. Hartle and P. Greenspan (2010). "Antiinflammatory activity of select sorghum (Sorghum bicolor) brans." J Med Food 13(4): 879-887.

Burdette, A., P. L. Garner, E. P. Mayer, J. L. Hargrove, D. K. Hartle and P. J. J. o. m. f. Greenspan (2010). "Anti-inflammatory activity of select sorghum (Sorghum bicolor) brans." 13(4): 879-887.

Cantin, C. M., M. A. Moreno and Y. Gogorcena (2009). "Evaluation of the antioxidant capacity, phenolic compounds, and vitamin $\mathrm{C}$ content of different peach and nectarine [ Prunus persica (L.) Batsch] breeding progenies." J Agric Food Chem 57(11): 4586-4592.

Chandra, D., S. Chandra and A. Sharma (2016). "Review of Finger millet (Eleusine coracana (L.) Gaertn): a power house of health benefiting nutrients." J Food Science Human Wellness 5(3): 149-155.

Chen, P., F. Chen and B. Zhou (2018). "Antioxidative, anti-inflammatory and anti-apoptotic effects of ellagic acid in liver and brain of rats treated by D-galactose." Sci Rep 8(1): 1465.

Chethan, S. and N. J. A. J. o. F. T. Malleshi (2007). "Finger millet polyphenols: Characterization and their nutraceutical potential." 2(7): 582-592.

Clark, J. D., A. R. Schievella, E. A. Nalefski and L. L. Lin (1995). "Cytosolic phospholipase A2." J Lipid Mediat Cell Signal 12(2-3): 83-117.

Conner, E. M. and M. B. Grisham (1996). "Inflammation, free radicals, and antioxidants." Nutrition 12(4): 274277.

Davidson, F., E. Dennis, M. Powell and J. Glenney Jr (1987). "Inhibition of phospholipase A2 by" lipocortins" and calpactins. An effect of binding to substrate phospholipids." J Biol Chem 262(4): 1698-1705.

Dillard, C. J. and J. B. German (2000). "Phytochemicals: nutraceuticals and human health." J Science of Food and Agriculture 80(12): 1744-1756.

Elford, P. R., M. Graeber, H. Ohtsu, M. Aeberhard, B. Legendre, W. L. Wishart and A. R. MacKenzie (1992). "Induction of swelling, synovial hyperplasia and cartilage proteoglycan loss upon intra-articular injection of transforming growth factor $\beta-2$ in the rabbit." J Cytokine 4(3): 232-238.

Forni, C., F. Facchiano, M. Bartoli, S. Pieretti, A. Facchiano, D. D’Arcangelo, S. Norelli, G. Valle, R. Nisini and S. J. B. r. i. Beninati (2019). "Beneficial role of phytochemicals on oxidative stress and age-related diseases." 2019.

Francis, B., C. Seebart and Kaiser, II (1992). "Citrate is an endogenous inhibitor of snake venom enzymes by metal-ion chelation." Toxicon 30(10): 1239-1246.

Franson, R., R. Raghupathi, M. Fry, J. Saal, B. Vishwanath, S. S. Ghosh and M. D. Rosenthal (1990). "Inhibition of human phospholipases A2 by cis-unsaturated fatty acids and oligomers of prostaglandin B1." $\underline{\mathrm{Adv}}$ Exp Med Biol 279: 219-230.

Gijón, M. A. and C. C. Leslie (1999). "Regulation of arachidonic acid release and cytosolic phospholipase A2 activation." J Leukocyte Biology 65(3): 330-336.

Hao, S., K. Chen, L. Cao, X. Zhu, G. Luo, S. Zhang and J. Chen (2016). "Separation of high-purity syringol and acetosyringone from rice straw-derived bio-oil by combining the basification-acidification process and column chromatography." Electrophoresis 37(19): 2522-2530.

Intisar, A., L. Zhang, H. Luo, J. B. Kiazolu, R. Zhang and W. Zhang (2012). "Anticancer constituents and cytotoxic activity of methanol-water extract of Polygonum bistorta L." $\underline{\text { Afr J Tradit Complement Altern Med }}$ 10(1): 53-59.

Jameel, N. M., B. M. Frey, F. J. Frey, T. V. Gowda and B. S. Vishwanath (2005). "Inhibition of secretory phospholipase $\mathrm{A}(2)$ enzyme by bilirubin: a new role as endogenous anti-inflammatory molecule." Mol Cell Biochem 276(1-2): 219-225. 
Jameel, N. M., M. A. Shekhar and B. S. Vishwanath (2006). " $\alpha$-lipoic acid: An inhibitor of secretory phospholipase A2 with anti-inflammatory activity." Life Sciences 80(2): 146-153.

Jensen, W. B. (2007). "The origin of the Soxhlet extractor." J Chem Educ 84(12): 1913.

Joshi, V., M. Umashankara, C. Ramakrishnan, A. N. Nanjaraj Urs, K. N. Suvilesh, D. Velmurugan, K. S. Rangappa and B. S. Vishwanath (2016). "Dimethyl ester of bilirubin exhibits anti-inflammatory activity through inhibition of secretory phospholipase A2, lipoxygenase and cyclooxygenase." Arch Biochem Biophys 598: 2839.

Joshi, V., S. H. Venkatesha, C. Ramakrishnan, A. N. Nanjaraj Urs, V. Hiremath, K. D. Moudgil, D. Velmurugan and B. S. Vishwanath (2016). "Celastrol modulates inflammation through inhibition of the catalytic activity of mediators of arachidonic acid pathway: Secretory phospholipase A2 group IIA, 5-lipoxygenase and cyclooxygenase-2." Pharmacol Res 113(Pt A): 265-275.

Jun, H. I., G. S. Song, E. I. Yang, Y. Youn and Y. S. Kim (2012). "Antioxidant activities and phenolic compounds of pigmented rice bran extracts." J Food science 77(7): C759-C764.

Kozuka, C., S. Sunagawa, R. Ueda, M. Higa, Y. Ohshiro, H. Tanaka, C. Shimizu-Okabe, C. Takayama, M. Matsushita, M. Tsutsui, S. Ishiuchi, M. Nakata, T. Yada, J. I. Miyazaki, S. Oyadomari, M. Shimabukuro and H. Masuzaki (2015). "A novel insulinotropic mechanism of whole grain-derived gamma-oryzanol via the suppression of local dopamine D2 receptor signalling in mouse islet." Br J Pharmacol 172(18): 4519-4534.

Krzyzanowska, J., A. Czubacka and W. Oleszek (2010). "Dietary phytochemicals and human health." Farms for Nutraceuticals: 74-98.

Kumar, A., M. Metwal, S. Kaur, A. K. Gupta, S. Puranik, S. Singh, M. Singh, S. Gupta, B. K. Babu and S. Sood (2016). "Nutraceutical value of finger millet [Eleusine coracana (L.) Gaertn.], and their improvement using omics approaches." Frontiers in plant science 7: 934.

Laine, L. (2003). "Gastrointestinal effects of NSAIDs and coxibs." J Pain Symptom Management 25(2): 32-40. Larrosa, M., M. J. Yañéz-Gascón, M. a. V. Selma, A. Gonzalez-Sarrias, S. Toti, J. J. Cerón, F. Tomas-Barberan, P. Dolara and J. C. Espín (2009). "Effect of a low dose of dietary resveratrol on colon microbiota, inflammation and tissue damage in a DSS-induced colitis rat model." J Agricultural Food Chemistry 57(6): 2211-2220.

Lawrence, T. (2009). "The nuclear factor NF-kappaB pathway in inflammation." Cold Spring Harb Perspect Biol 1(6): a001651.

Lee, W. H., M. H. Toyama, A. M. Soares, J. R. Giglio, S. Marangoni and I. Polikarpov (1999). "Crystallization and preliminary X-ray diffraction studies of piratoxin III, a D-49 phospholipase A2 from the venom of Bothrops pirajai." Acta Crystallogr D Biol Crystallogr 55(Pt 6): 1229-1230.

Li, Y., L. Zhang, X. Wang, W. Wu and R. Qin (2019). "Effect of Syringic acid on antioxidant biomarkers and associated inflammatory markers in mice model of asthma." Drug Dev Res 80(2): 253-261.

Liu, Y., H. Tang, X. Liu, H. Chen, N. Feng, J. Zhang, C. Wang, M. Qiu, J. Yang and X. Zhou (2019). "Frontline Science: Reprogramming COX-2, 5-LOX, and CYP4A-mediated arachidonic acid metabolism in macrophages by salidroside alleviates gouty arthritis." J Leukoc Biol 105(1): 11-24.

Loo, A., K. Jain and Darah (2008). "Antioxidant activity of compounds isolated from the pyroligneous acid, Rhizophora apiculata." J Food chemistry 107(3): 1151-1160.

Lowry, O. H., N. J. Rosebrough, A. L. Farr and R. J. Randall (1951). "Protein measurement with the Folin phenol reagent." J Biol Chem 193(1): 265-275.

Luitel, D. R., M. Siwakoti and P. K. Jha "Eleusine coracana (L) Gaertn (Finger millet): A Crop With Medicinal Value."

Maga, J. A. (1987). "The flavor chemistry of wood smoke." J Food Reviews International 3(1-2): 139-183.

Medzhitov, R. (2010). "Inflammation 2010: new adventures of an old flame." Cell 140(6): 771-776.

Mirzoeva, O. and P. Calder (1996). "The effect of propolis and its components on eicosanoid production during the inflammatory response." J Prostaglandins, Leukotrienes Essential Fatty Acids 55(6): 441-449.

Monteseirín, J., I. Bonilla, J. Camacho, J. Conde and F. Sobrino (2001). "Elevated secretion of myeloperoxidase by neutrophils from asthmatic patients: the effect of immunotherapy." J Allergy Clinical Immunology 107(4): 623-626.

Mourits, M. P., M. F. Prummel, W. M. Wiersinga and L. J. C. e. Koornneef (1997). "Clinical activity score as a guide in the management of patients with Graves' ophthalmopathy." 47(1): 9-14.

Nanjaraj Urs, A. N., M. Yariswamy, V. Joshi, K. N. Suvilesh, M. S. Sumanth, D. Das, A. Nataraju and B. S. Vishwanath (2015). "Local and systemic toxicity of Echis carinatus venom: neutralization by Cassia auriculata L. leaf methanol extract." J Nat Med 69(1): 111-122.

Pruzanski, W., R. A. Greenwald, L. P. Street, F. Lauberte, E. Stefanski and P. Vadas (1992). "Inhibition of enzymatic activity of phospholipases A2 by minocycline and doxycycline." J Biochemical pharmacology 44(6): 1165-1170.

Ramazani, E., M. Akaberi, S. A. Emami and Z. Tayarani-Najaran (2020). "Biological and pharmacological effects of gamma-oryzanol: an updated review of the molecular mechanisms." Curr Pharm Des. 
Rao, C. V., A. Rivenson, B. Simi and B. S. J. C. r. Reddy (1995). "Chemoprevention of colon carcinogenesis by dietary curcumin, a naturally occurring plant phenolic compound." 55(2): 259-266.

Rao, C. V. J. T. m. t., t. u. o. c. i. health and disease (2007). "Regulation of COX and LOX by curcumin." 213226.

Rasouli, H., M. H. Farzaei and R. Khodarahmi (2017). "Polyphenols and their benefits: A review." J International Journal of Food Properties 20(sup2): 1700-1741.

Savitha Prashanth, M. R., R. R. Shruthi and G. Muralikrishna (2015). "Immunomodulatory activity of purified arabinoxylans from finger millet (Eleusine coracana, v. Indaf 15) bran." J Food Sci Technol 52(9): 6049-6054.

Schieven, G. L. (2005). "The biology of p38 kinase: a central role in inflammation." J Current topics in medicinal chemistry 5(10): 921-928.

Sharma, H. S., J. Westman, F. Nyberg, J. Cervos-Navarro and P. Dey (1994). Role of serotonin and prostaglandins in brain edema induced by heat stress. An experimental study in the young rat. Brain edema ix: 65-70.

Somintara, S., V. Leardkamolkarn, P. Suttiarporn and S. Mahatheeranont (2016). "Anti-tumor and immune enhancing activities of rice bran gramisterol on acute myelogenous leukemia." J PLoS One 11(1): e0146869.

Srivastava, K., A. J. T. E. J. o. P. S. Sharma and Biotechnology (2012). "Nutraceutical importance of finger millet (Eleusine coracana) for improved human health." $6(\mathrm{~S}(2))$.

Suram, S., T. A. Gangelhoff, P. R. Taylor, M. Rosas, G. D. Brown, J. V. Bonventre, S. Akira, S. Uematsu, D. L. Williams, R. C. Murphy and C. C. Leslie (2010). "Pathways regulating cytosolic phospholipase A2 activation and eicosanoid production in macrophages by Candida albicans." J Biol Chem 285(40): 30676-30685.

Suttiarporn, P., W. Chumpolsri, S. Mahatheeranont, S. Luangkamin, S. Teepsawang and V. Leardkamolkarn (2015). "Structures of phytosterols and triterpenoids with potential anti-cancer activity in bran of black nonglutinous rice." Nutrients 7(3): 1672-1687.

Tarayre, J. P. and H. Lauressergues (1979). "Comparison of the effects of a non-steroidal anti-inflammatory agent, an immunosuppressive, a corticosteroid and an immunomodulator on various immunological and nonimmunological inflammatory experimental models." Arch Int Pharmacodyn Ther 242(1): 159-176.

Toyama, M. H., C. R. Costa, M. N. Belchor, D. P. Novaes, M. A. de Oliveira, R. Ie, H. H. Gaeta and D. d. O. Toyama (2019). Edema Induced by sPLA2 from Crotalus durissus terrificus involves PLC and PKC signaling, activation of cPLA2, and Oxidative Stress. Edema-Diagnosis and Treatment.

Verma, A. K. and S. Singh (2020). "Phytochemical analysis and in vitro cytostatic potential of ethnopharmacological important medicinal plants." Toxicol Rep 7: 443-452.

Vishwanath, B. S., A. A. Fawzy and R. C. Franson (1988). "Edema-inducing activity of phospholipase A2 purified from human synovial fluid and inhibition by aristolochic acid." Inflammation 12(6): 549-561.

Wang, J., Z. Guo, Y. Fu, Z. Wu, C. Huang, C. Zheng, P. A. Shar, Z. Wang, W. Xiao and Y. Wang (2017). "Weak-binding molecules are not drugs?-toward a systematic strategy for finding effective weak-binding drugs." Brief Bioinform 18(2): 321-332.

Wang, S.-H. (2007). Pharmaceutical composition containing guaiacol derivatives and syringol derivatives extracted from natural plant vinegar, Google Patents.

Yahfoufi, N., N. Alsadi, M. Jambi and C. Matar (2018). "The immunomodulatory and anti-inflammatory role of polyphenols." J Nutrients 10(11): 1618.

Yedgar, S., D. Lichtenberg and E. Schnitzer (2000). "Inhibition of phospholipase A(2) as a therapeutic target." Biochim Biophys Acta 1488(1-2): 182-187.

Yin, Z. N., W. J. Wu, C. Z. Sun, H. F. Liu, W. B. Chen, Q. P. Zhan, Z. G. Lei, X. Xin, J. J. Ma, K. Yao, T. Min, M. M. Zhang and H. Wu (2019). "Antioxidant and Anti-inflammatory Capacity of Ferulic Acid Released from Wheat Bran by Solid-state Fermentation of Aspergillus niger." Biomed Environ Sci 32(1): 11-21.

Zhu, F., B. Du, B. J. C. r. i. f. s. Xu and nutrition (2018). "Anti-inflammatory effects of phytochemicals from fruits, vegetables, and food legumes: A review." 58(8): 1260-1270.

Zhu, L., P. Gu and H. Shen (2019). "Gallic acid improved inflammation via NF-kappaB pathway in TNBSinduced ulcerative colitis." Int Immunopharmacol 67: 129-137. 
Graphical abstract:
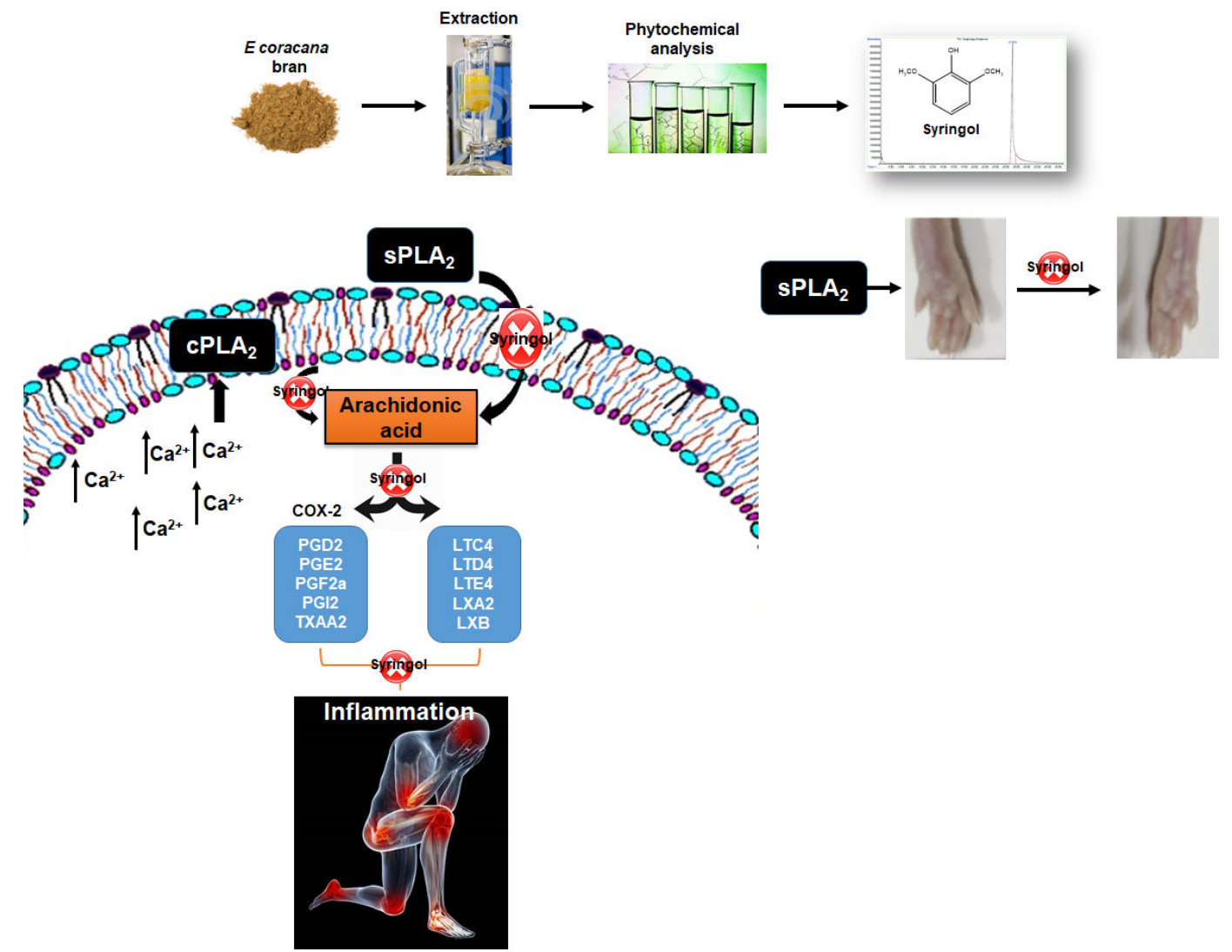